\title{
HÖLDER REGULARITY FOR THE TIME FRACTIONAL SCHRÖDINGER EQUATION
}

\author{
XIAOYAN SU AND JIQIANG ZHENG
}

\begin{abstract}
In this paper, we investigate the Hölder regularity of solutions to the time fractional Schrödinger equation of order $1<\alpha<2$, which interpolates between the Schrödinger and wave equations. This is inspired by Hirata and Miao's work [10] which studied the fractional diffusion-wave equation. First, we give the asymptotic behavior for the oscillatory distributional kernels and their Bessel potentials by using Fourier analytic techniques. Then, the space regularity is derived by employing some results on singular Fourier multipliers. Using the asymptotic behavior for the above kernels, we prove the time regularity. Finally, we use mismatch estimates to prove the pointwise convergence to the initial data in Hölder spaces. In addition, we also prove Hölder regularity result for the Schrödinger equation.
\end{abstract}

Key Words: Time fractional Schrödinger equation; Hölder regularity; singular Fourier multipliers; pointwise convergence in Hölder space.

AMS Classification: 35R11, 42B15, 35A09

\section{INTRODUCTION}

In this paper, we investigate the Hölder regularity of solutions to the following Cauchy problem,

$$
\left\{\begin{array}{l}
i^{\alpha} D_{t}^{\alpha} u(t, x)=-\Delta u(t, x)+f(t, x), \quad(t, x) \in \mathbb{R}^{+} \times \mathbb{R}^{n}, \\
u(0, x)=u_{0}(x), \partial_{t} u(0, x)=u_{1}(x), \quad x \in \mathbb{R}^{n},
\end{array}\right.
$$

where $D_{t}^{\alpha}$ is the Caputo derivative of order $1<\alpha<2$, which interpolates between the Schrödinger equation $(\alpha=1)$ and wave equation $(\alpha=2)$.

Time fractional differential equations have recently become a topic of active research. Many of these papers study the time fractional generalization of the heat equation or the wave equation, with form

$$
D_{t}^{\alpha} u(t, x)=\Delta u(t, x)+f(t, x), \quad(t, x) \in \mathbb{R}^{+} \times \mathbb{R}^{n},
$$

which we will refer as 'diffusion-wave' equations. For example, the fundamental solutions of the diffusion-wave equations have been given in [19, 30] and the asymptotic expansions of the fundamental solutions have been fully developed in [1, 15]. The $L_{t}^{p} L_{x}^{q}$ theory for time fractional evolution equations with variable coefficients has been established in 14. Some probabilistic methods have been used in [6, (7) to study these diffusion-wave equations. Also, see [10, 21, 22] for more topics.

The time fractional Schrödinger equation has been studied in [4, 9, 18, 26, 32, 33, but our equation is different because they restrict the order $\alpha$ of the time fractional derivative to the range $(0,1)$. In contrast, we consider here the time fractional derivative $D_{t}^{\alpha}$ of order $\alpha \in(1,2)$, which has not been considered in the previously mentioned papers. In addition, in our equation (1.1), the term $D_{t}^{\alpha} u$ is multiplied by $i^{\alpha}$, which makes it different from the diffusion-wave equation (1.2). Also, when 
$\alpha=1$, the equation (1.1) is the classical Schrödinger equation, and when $\alpha=2$, the equation (1.1) is the wave equation.

Using the Fourier-Laplace transform, it is easy to solve the Cauchy problem (1.1) in the sense of mild solutions (see Definition 2.13 below). In this paper, we are interested in the minimal regularity required to understand (1.1) in a pointwise sense. Intuitively, all the terms in the equation make sense pointwise as long as $u$ is $C_{t}^{\alpha}$ in time (see Definition 2.2 below) and $C^{2}$ in space. So the question becomes: what regularity should we impose on the initial data $\left(u_{0}, u_{1}\right)$ so that the mild solution is a classical solution in the sense of Definition 2.15?

To answer this question, we can first look at the endpoint cases $\alpha \in\{1,2\}$. For the wave equation $(\alpha=2)$, it is well known that in order to make sure that the solution is classical ( $C^{2}$ in both space and time), the initial data should belong to $C^{[n / 2]+2}\left(\mathbb{R}^{n}\right)$. So heuristically, the solution loses $[n / 2]$ 'derivatives'. This result can be obtained either by giving an explicit formula for the solution (see Evan's [5] or Miao's [22]) or by investigating the order of the fundamental solution as a distribution, as in Hörmander's book [11.

For the Schrödinger equation $(\alpha=1)$, to the best of the authors' knowledge, classical solutions of this Hölder regularity has not been studied, since it is more natural from physical considerations to study this equation with $L^{2}$ based Sobolev spaces. However, we can still ask similar question for the Schrödinger equation: what is the minimal regularity can guarantee the existence of the classical solution? To answer this question, we also prove a new result for the classical Schrödinger equation (see Theorem 1.2 below).

The existence of classical solutions for the diffusion-wave equation (1.2) is proven by studying the asymptotic behavior of the fundamental solution using Fox $\mathrm{H}$ functions, see [12, 16, 17, 28. However, we cannot adapt this method to study (1.1) because in our case, the variable $z=i^{-\alpha} x t^{-\alpha / 2}$ lies in a critical line $|\arg z|=\frac{\alpha \pi}{2}$ of the corresponding $H$-functions and the asymptotic behavior on this line cannot be given simply by analyzing the residues of $H$-functions.

Instead, we will use Fourier analysis techniques to study (1.1) for $\alpha \in(1,2)$. In contrast to the fundamental solutions for the diffusion-wave equations which have polynomial decay as $|z| \rightarrow \infty$, we prove (see Lemma 3.1 below) that the distributional kernels for (1.1) are oscillating without decay as $|z| \rightarrow \infty$. This motivates the study of Fourier multipliers related to (1.1) in Hölder spaces, which allow us to prove the space regularity of solutions. To obtain the time regularity, we prove some upper bounds for the Bessel potentials of the corresponding kernels to verify the exchange of limits and integrals.

Finally, we prove that the initial data is attained in a pointwise sense. As it is well known, this is easy to prove for the wave equation since the fundamental solution is compactly supported, a property also known as the finite speed of propagation. For the Schrödinger equation, finite speed of propagation is not available which complicates the proof. As a replacement, we use 'almost finite speed of propagation', also known as 'mismatch estimates' in the literature (see [13). For (1.1) with $\alpha \in(1,2)$, it turns out that analogous mismatch estimates are also true, which allow us to prove that if the initial data belongs to a certain Hölder space, then solutions to (1.1) with $\alpha \in(1,2)$ pointwise attain the initial data.

Our main results are as follows: 
Theorem 1.1. For $1<\alpha<2$ and any $\epsilon>0$, if $u_{0} \in C^{2+\frac{n}{\alpha}+\epsilon}\left(\mathbb{R}^{n}\right), u_{1} \in$ $C^{2+\frac{n}{\alpha}-\frac{2}{\alpha}+\epsilon}\left(\mathbb{R}^{n}\right)$ and $f \in C_{t}^{0}\left((0, \infty) ; C^{\frac{n}{\alpha}+\frac{2}{\alpha}+\epsilon}\left(\mathbb{R}^{n}\right)\right)$, then the mild solution $u(t, x)$ defined by (2.13) is a classical solution defined in (2.15).

For equation (1.1) with $\alpha=1$, which is the Schrödinger equation, we only need to prescribe $u_{0}$, that is

$$
\left\{\begin{array}{lrl}
i \partial_{t} u(t, x)=-\Delta u(t, x)+f(t, x), & (t, x) & \in \mathbb{R}^{+} \times \mathbb{R}^{n}, \\
u(0, x)=u_{0}(x), & x & \in \mathbb{R}^{n},
\end{array}\right.
$$

and we have the following result:

Theorem 1.2. For $\alpha=1$ and any $\epsilon>0$, if $u_{0}(x) \in C^{n+2+\epsilon}\left(\mathbb{R}^{n}\right)$ and $f \in$ $C_{t}^{0}\left((0, \infty) ; C^{n+\epsilon}\left(\mathbb{R}^{n}\right)\right)$, then the mild solution defined in (5.1) is a classical solution .

Remark 1.3. If we ignore the small $\epsilon$, the Theorem 1.1 and 1.2 are sharp from the perspective of Fourier multiplier results. However, using the methods in this paper, we need a small extra $\epsilon$ to guarantee the existence of classical solution. Whether we can remove this $\epsilon$ is unclear at this moment.

Remark 1.4. Although the problem in this paper is inspired by the wave equation, the methods used here cannot be applied to recover the known results for the wave equation. The main reason is that the Fourier multiplier $e^{i|\xi|^{\frac{2}{\alpha}}}$ for $\alpha=2$ behaves quite differently with the multiplier $1<\alpha<2$. The corresponding kernel for the former has singularity at $|x|=1$, whereas the singular points for the latter appears only at $|x|=0$ or $|x|=\infty$, 25, for the details.

This paper is organized as follows. Some preliminaries are given in section 2. In section 3, we derive the asymptotic behavior for the oscillatory kernel functions, and some delicate upper bounds for their Bessel potentials are obtained by using Fourier analysis methods. In subsection 4.1, we explore the Fourier multiplier properties for the oscillatory Mittag-Leffler functions and prove the space regularity in Hölder spaces. In subsection 4.2, the time regularity is proved using the estimates for the Bessel potentials of the corresponding kernels. In subsection 4.3, we prove the pointwise convergence to the initial data by using the almost finite speed propagation property for the solutions. The Schrödinger equation will be discussed in section 5 .

\section{Preliminaries}

In this section, we will review some basic definitions and properties about the fractional derivatives, Mittag-Leffler functions, Holder spaces and Besov spaces and closely related Bessel potentials. Some results on singular Fourier multipliers are also included. Also we recall one version Faá di Bruno's formula which will be used in our paper later. Finally, the classical solutions and mild solutions are given using the notations introduced above.

We denote by $\mathcal{S}$ the Schwartz space on $\mathbb{R}^{n}$ and by $\mathcal{S}^{\prime}$ of temper distributions. The Fourier transform and the inverse Fourier transform are defined by

$$
\mathcal{F}(f)(\xi)=\widehat{f}(\xi)=\frac{1}{(2 \pi)^{n / 2}} \int_{\mathbb{R}^{n}} e^{-i \xi \cdot x} f(x) \mathrm{d} x,
$$


and

$$
\mathcal{F}^{-1}(f)(x)=\frac{1}{(2 \pi)^{n / 2}} \int_{\mathbb{R}^{n}} e^{i x \cdot \xi} f(\xi) \mathrm{d} \xi .
$$

For a function $m(\xi)$, we can associate it with an operator $T_{m}$ given by

$$
T_{m} f(x)=\frac{1}{(2 \pi)^{n / 2}} \int_{\mathbb{R}^{n}} e^{i x \cdot \xi} m(\xi) \widehat{f}(\xi) \mathrm{d} \xi .
$$

We say that ' $m(\xi)$ is a multiplier from $X$ to $Y$ ' if $T_{m}$ is a bounded operator from $X$ to $Y$.

2.1. Fractional derivatives and properties. In this subsection, we review some definitions and properties about fractional derivatives, see [27.

For $\alpha>0$, we denote $g_{\alpha}(t)$ for

$$
g_{\alpha}(t):= \begin{cases}\frac{t^{\alpha-1}}{\Gamma(\alpha)}, & t>0, \\ 0, & t \leq 0,\end{cases}
$$

where $\Gamma(\alpha)$ is Gamma function. When $\alpha=0$, we denote $g_{0}(t)=\delta(t)$. And the Riemann-Liouville fractional integral of oder $\alpha \geq 0$ is defined as follows:

$$
I_{t}^{\alpha} f(t):=\left(g_{\alpha} * f\right)(t) .
$$

Let us use $\lceil\alpha\rceil$ to denote the smallest integer greater than or equal to $\alpha$. Then the Riemann-Liouville fractional derivative of order $\alpha>0$ is given by

$$
\partial_{t}^{\alpha} f(t)=D_{t}^{\lceil\alpha\rceil} I_{t}^{\lceil\alpha\rceil-\alpha} f(t),
$$

where $D_{t}^{m}=\frac{d^{m}}{d t^{m}}$ is the classical $m$ th derivative.

Generally, the Caputo derivative of order $\alpha>0$ is given by

$$
D_{t}^{\alpha} f(t)=I_{t}^{\lceil\alpha\rceil-\alpha} D_{t}^{\lceil\alpha\rceil} f(t) .
$$

From the above definitions, it is obvious that we need $f(t)$ to be $C^{\lceil\alpha\rceil}$-times differentiable to define the Caputo derivatives. Whereas, less regularity is required to define its Riemann-Liouville derivatives.

In addition, it is easy to check that the following equation is valid for smooth enough function $f(t)$ using integration by parts,

$$
\left.D_{t}^{\alpha} f(t)=\partial_{t}^{\alpha}\left(f(t)-\sum_{k=0}^{\lceil\alpha\rceil-1} f^{(k}\right)(0) g_{k+1}(t)\right) .
$$

Therefore, we can redefine the Caputo derivative using Riemann-Liouville fractional derivative according to (2.2) to lower the regularity for $f(t)$.

Applying the properties of the Laplace transform, we have

$$
\begin{aligned}
\mathcal{L}\left(\partial_{t}^{\alpha} f(t)\right)(\lambda) & =\lambda^{\alpha} \mathcal{L}(f(t))-\sum_{k=0}^{\lceil\alpha\rceil-1}\left(g_{\lceil\alpha\rceil-\alpha} * f\right)^{(k)}(0) \lambda^{\lceil\alpha\rceil-1-k}, \\
\mathcal{L}\left(D_{t}^{\alpha} f(t)\right)(\lambda) & =\lambda^{\alpha} \mathcal{L}(f(t))-\sum_{k=0}^{\lceil\alpha\rceil-1} f^{(k)}(0) \lambda^{\lceil\alpha\rceil-1-k} .
\end{aligned}
$$

where $\mathcal{L}$ denotes the Laplace transform. As we can see from the above equalities, the Laplace transform for the Caputo derivative is simpler than the RiemannLiouville derivative, that is part of the reason why we choose the Caputo fractional derivatives in our paper. 
To be more clear, we give the definition $D_{t}^{\alpha}$ for $1<\alpha<2$ in the following:

Definition 2.1. Let $1<\alpha<2$, using (2.2) we define the fractional Caputo derivative of a function $f(t)$ by

$$
D_{t}^{\alpha} f(t)=D_{t}^{2}\left(\frac{1}{\Gamma(2-\alpha)} \int_{0}^{t}(t-\tau)^{1-\alpha}\left(f(\tau)-f(0)-f^{\prime}(0) \tau\right) \mathrm{d} \tau\right),
$$

For the above definition, we have

$$
\mathcal{L}\left(D_{t}^{\alpha} f(t)\right)(\lambda)=\lambda^{\alpha} \mathcal{L}(f(t))-\lambda^{\alpha-1} f(0)-\lambda^{\alpha-2} f^{\prime}(0) .
$$

Now we introduce time Hölder space which closely related to the fractional Caputo derivatives above, and it is different with the spatial Hölder spaces which will be introduced later.

Definition 2.2. We use $C_{t}^{0}(0, \infty)$ to denote the set of all the continuous and uniformly bounded functions with respect to $t>0$. For $1<\alpha<2$, we say a function $f(t) \in C_{t}^{\alpha}(0, \infty)$ if $g_{2-\alpha} * f(t) \in C^{2}(0, \infty)$.

2.2. Mittag-Leffler functions. In this subsection, we introduce the MittagLeffler functions, its asymptotic behaviors, and its derivatives. More information about Mittag-Leffler functions can be found in [8].

Definition 2.3. [8, p.56] The two parameter Mittag-Leffler functions are given by

$$
E_{\alpha, \beta}(z)=\sum_{k=0}^{\infty} \frac{z^{k}}{\Gamma(\alpha k+\beta)}, \quad(\alpha>0, \beta \in \mathbb{C}) .
$$

The most interesting properties of the Mittag-Leffler functions are associated with their Laplace transforms,

$$
\mathcal{L}\left(t^{\beta-1} E_{\alpha, \beta}\left(\omega t^{\alpha}\right)(\lambda)=\int_{0}^{\infty} e^{-\lambda t} t^{\beta-1} E_{\alpha, \beta}\left(\omega t^{\alpha}\right) \mathrm{d} t=\frac{\lambda^{\alpha-\beta}}{\lambda^{\alpha}-\omega} .\right.
$$

In the following, we give the asymptotic behavior of Mittag-Leffler functions.

Theorem 2.4. [8, p.64 Theorem 4.3] For $0<\alpha<2, \beta \in \mathbb{C}, m \in \mathbb{N}$, the following asymptotic formulas hold:

(1) For any $z \in \mathbb{C}$, we have

$$
E_{\alpha, \beta}(z)=\sum_{k=0}^{m} \frac{z^{k}}{\Gamma(\alpha k+\beta)}+O\left(|z|^{m+1}\right), \quad|z| \rightarrow 0 .
$$

(2) If $|\arg z|<\min \{\pi, \alpha \pi\}$, then

$$
E_{\alpha, \beta}(z)=\frac{1}{\alpha} z^{(1-\beta) / \alpha} \mathrm{e}^{z^{1 / \alpha}}-\sum_{k=1}^{m} \frac{z^{-k}}{\Gamma(\beta-k \alpha)}+O\left(|z|^{-m-1}\right), \quad|z| \rightarrow \infty .
$$

In particular, when $|z| \geq 1$, with $|\arg z|=\frac{\pi}{2}$, then we have

$$
\left|z^{\beta-1} E_{\alpha, \beta}\left(z^{\alpha}\right)\right| \leq C \text {. }
$$

The next theorem deals with the derivatives of the Mittag-Leffler functions.

Theorem 2.5. [8, p.58] Let $m \geq 1$ be an integer, then

$$
\frac{d^{m}}{d z^{m}}\left[z^{\beta-1} E_{\alpha, \beta}\left(z^{\alpha}\right)\right]=z^{\beta-m-1} E_{\alpha, \beta-m}\left(z^{\alpha}\right), \quad(m \geq 1) .
$$


2.3. Hölder spaces and Besov spaces. In this subsection, we will recall the Hölder spaces and Besov spaces in $\mathbb{R}^{n}$, see [29].

Let $\gamma=\left(\gamma_{1}, \cdots, \gamma_{n}\right)$ be a multi-index of non-negative integers, and $|\gamma|=\gamma_{1}+$ $\cdots+\gamma_{n}$, then denote

$$
D^{\gamma} f(x)=\frac{\partial^{|\gamma|} f(x)}{\partial x_{1}^{\gamma_{1}} \cdots \partial x_{n}^{\gamma_{n}}} .
$$

Definition 2.6. ([29, p.36] Hölder spaces $\left.C^{s}\left(\mathbb{R}^{n}\right)\right)$. We use $C^{0}\left(\mathbb{R}^{n}\right)=C\left(\mathbb{R}^{n}\right)$ to denote the set of all complex-valued bounded and uniformly continuous functions on $\mathbb{R}^{n}$. When $s \geq 0$ is an integer,

$$
\begin{aligned}
C^{s}\left(\mathbb{R}^{n}\right) & =\left\{f: \mathbb{R}^{n} \rightarrow \mathbb{C} \mid D^{\gamma} f \in C\left(\mathbb{R}^{n}\right), \quad \text { for all }|\gamma| \leq s\right\}, \text { with } \\
\|f\|_{C^{s}\left(\mathbb{R}^{n}\right)} & =\sum_{|\gamma| \leq s}\left\|D^{\gamma} f\right\|_{C\left(\mathbb{R}^{n}\right)} .
\end{aligned}
$$

If $s>0$ is not an integer, then we write $s=[s]+\{s\}$ with $[s]$ integer, and $0<$ $\{s\}<1$, then

$$
\begin{aligned}
C^{s}\left(\mathbb{R}^{n}\right) & =\left\{f: \mathbb{R}^{n} \rightarrow \mathbb{C} \mid f \in C^{[s]}\left(\mathbb{R}^{n}\right),\|f\|_{C^{s}\left(\mathbb{R}^{n}\right)}<\infty\right\}, \text { with } \\
\|f\|_{C^{s}\left(\mathbb{R}^{n}\right)} & =\|f\|_{C^{[s]}\left(\mathbb{R}^{n}\right)}+\sum_{|\gamma|=[s]} \sup _{x \neq y} \frac{\left|D^{\gamma} f(x)-D^{\gamma} f(y)\right|}{|x-y|^{\{s\}}}
\end{aligned}
$$

In this paper, we use $C_{t}^{0}\left((0, \infty) ; C^{\theta}\left(\mathbb{R}^{n}\right)\right)$ to denote all the functions $f(t, x)$ such that $\|f(t, x)\|_{C^{\theta}\left(\mathbb{R}^{n}\right)} \in C_{t}^{0}(0, \infty)$.

Definition 2.7. ([29, p.45] Inhomogeneous Besov space) Let $\left\{\psi_{j}\right\}_{j \geq 0} \subset \mathcal{S}\left(\mathbb{R}^{n}\right)$ be a family of functions such that

$$
\left\{\begin{array}{l}
\operatorname{supp} \psi_{0} \subset\{x|| x \mid \leq 2\}, \\
\operatorname{supp} \psi_{j} \subset\left\{x\left|2^{j-1} \leq\right| x \mid \leq 2^{j}\right\}, j \geq 1,
\end{array}\right.
$$

for every multi-index $\gamma$ there exists a positive number $c_{\gamma}$ such that $2^{j|\gamma|}\left|D^{\gamma} \psi_{j}(x)\right| \leq$ $c_{\gamma}$ for all $x \in \mathbb{R}^{n}$ and $\sum_{j=0}^{\infty} \psi_{j}(x)=1$ for every $x \in \mathbb{R}^{n}$.

The inhomogeneous Besov space is given by

$$
B_{p, q}^{s}\left(\mathbb{R}^{n}\right)=\left\{f \in \mathcal{S}^{\prime}\left(\mathbb{R}^{n}\right):\|f\|_{B_{p, q}^{s}\left(\mathbb{R}^{n}\right)}<\infty\right\}
$$

where

$$
\|f\|_{B_{p, q}^{s}}:= \begin{cases}\left(\sum_{j=0}^{\infty}\left(2^{j s}\left\|\left(\psi_{j} \hat{f}\right)^{\vee}\right\|_{L^{p}}\right)^{q}\right)^{1 / q}, & 1 \leq q<\infty, \\ \sup _{j \geq 0} 2^{j s}\left\|\left(\psi_{j} \hat{f}\right)^{\vee}\right\|_{L^{p}}, & q=\infty .\end{cases}
$$

According to the Lemma 1 in 24 we have the following 'Young's Theorem' in Hölder spaces given as:

Lemma 2.8. If $f \in C^{s}\left(\mathbb{R}^{n}\right)$ and $g \in B_{1, \infty}^{0}\left(\mathbb{R}^{n}\right)$, then $f * g \in C^{s}\left(\mathbb{R}^{n}\right)$. 
2.4. Bessel potentials. In this subsection, we introduce the Bessel potentials and its operator properties acting on the Hölder space.

Definition 2.9 (See [2]). For any $\theta \in \mathbb{R}$, the Bessel potential of order $\theta$ for a tempered distribution $f$ is defined by

$$
J^{\theta} f(x)=\mathcal{F}^{-1}\left(\left(1+|\xi|^{2}\right)^{-\theta / 2} \widehat{f}(\xi)\right)(x) .
$$

For $\theta>0$, the function $\left(1+|\xi|^{2}\right)^{-\theta / 2}$ is the Fourier transform of a integrable function $G^{\theta}(x)$ where

$$
G^{\theta}(x)=\frac{1}{\Gamma(\theta / 2)} \int_{0}^{\infty} e^{-|x|^{2} / s} e^{-s / 4} s^{(-n+\theta) / 2-1} \frac{\mathrm{d} s}{s} .
$$

From a straightforward examination of the integral (2.9), we have for some $c>0$,

$$
\begin{array}{llrl}
G^{\theta}(x) & =O\left(e^{-c|x|}\right), & \text { as } & |x| \rightarrow \infty, \quad \text { and } \\
G^{\theta}(x)=\frac{|x|^{-n+\theta}}{\gamma(\theta)}+o\left(|x|^{-n+\theta}\right), & \text { as } \quad|x| \rightarrow 0,
\end{array}
$$

if $0<\alpha<n$.

The Bessel potential acts like a smoothing operator in Hölder space, in the following sense:

Proposition 2.10. [23, Theorem 6] For any $\theta \in \mathbb{R}, J^{\theta}$ maps $C^{s}\left(\mathbb{R}^{n}\right)$ isomorphically onto $C^{s+\theta}\left(\mathbb{R}^{n}\right)$.

2.5. Singular multipliers. In this subsection, we recall some known results on singular Fourier multipliers (see 25] for more details).

Let us denote by

$$
m_{a, b}^{ \pm}(\xi)=\phi(\xi)|\xi|^{-b} \exp \left( \pm i|\xi|^{a}\right), \xi \in \mathbb{R}^{n}, a>0, b \in \mathbb{R},
$$

where $\phi$ is a smooth function which vanishes in a neighborhood of the origin and equal to 1 outside a compact set. For this multiplier, we denote its kernel by $K_{a, b}^{ \pm}(x)=\mathcal{F}^{-1}\left(m_{a, b}^{ \pm}(\xi)\right)$. According to [25], we have the following property:

Theorem 2.11. 25, Proposition 5.1] When $a>1$ and $b \in \mathbb{R}, K_{a, b}^{+}$is smooth throughout $\mathbb{R}^{n}$, and

$$
K_{a, b}^{+}(x)=A|x|^{\frac{b-n+n a / 2}{1-a}} \exp \left(i B|x|^{-a /(1-a)}\right)+o\left(|x|^{\frac{b-n+n a / 2}{1-a}}\right), \quad \text { as } \quad|x| \rightarrow \infty,
$$

where $A, B$ are constants which depend on $a, b$ and $n$.

It is easy to notice that $K_{a, b}^{+}(x)$ and $K_{a, b}^{-}(x)$ are are complex conjugates of each other, thus they have the same asymptotic behavior.

In this paper, we will use the following inhomogeneous multiplier $\left(1+|\xi|^{2}\right)^{-b / 2}$ instead of homogeneous one $|\xi|^{-b}$, and we give the following corollary.

Corollary 2.12. If we denote by

$$
\tilde{m}_{a, b}^{ \pm}(\xi)=\phi(\xi)\left(1+|\xi|^{2}\right)^{-b / 2} \exp \left( \pm i|\xi|^{a}\right), \xi \in \mathbb{R}^{n}, a>0, b \in \mathbb{R},
$$

and $\tilde{K}_{a, b}^{ \pm}(x)=\mathcal{F}^{-1}\left(\tilde{m}_{a, b}^{ \pm}(\xi)\right)$, then $\tilde{K}_{a, b}^{ \pm}(x) \sim K_{a, b}^{ \pm}(x)$. To be precise, when $a>1$ and $b \in \mathbb{R}, \tilde{K}_{a, b}^{ \pm}(x)$ is smooth throughout $\mathbb{R}^{n}$, and

$$
\tilde{K}_{a, b}^{ \pm}(x)=A|x|^{\frac{b-n+n a / 2}{1-a}} \exp \left( \pm i B|x|^{-a /(1-a)}\right)+o\left(|x|^{\frac{b-n+n a / 2}{1-a}}\right), \quad \text { as } \quad|x| \rightarrow \infty,
$$

where $A, B$ are constants which depend on $a, b$ and $n$. 
Proof. Since we can write $\tilde{m}_{a, b}^{ \pm}=\frac{|\xi|^{b}}{\left(1+|\xi|^{2}\right)^{b / 2}} m_{a, b}^{ \pm}$. Then by Lemma 2, Chapter 4.3 in [31], $K_{b}(x):=\mathcal{F}^{-1}\left(\frac{|\xi|^{b}}{\left(1+|\xi|^{2}\right)^{b / 2}}\right)$ is a finite measure. Therefore, the property is valid.

Let $X$ and $Y$ be spaces of functions or distributions on $\mathbb{R}^{n}$, and define $\mathcal{M}(X, Y)$ to be the spaces of all $m \in \mathcal{S}^{\prime}$ such that $\|m\|_{\mathcal{M}(X, Y)}<\infty$, where

$$
\|m\|_{\mathcal{M}(X, Y)}=\sup \left\{\frac{\left\|\mathcal{F}^{-1}(m \widehat{f})\right\|_{Y}}{\|f\|_{X}}: f \in \mathcal{D} \cap X,\|f\|_{X} \neq 0\right\} .
$$

Theorem 2.13. 25, Theorem 5.1] Let $a>0, \theta \in \mathbb{R}$. Suppose that $A \geq 1, m$ is a function of class $C^{\left[\frac{n}{2}\right]+1}$ on $\mathbb{R}^{n}, m(\xi)=0$ for $|\xi| \leq 2$ and

$$
\left|D^{\gamma} m(\xi)\right| \leq|\xi|^{-\theta-n a / 2}\left(A|\xi|^{a-1}\right)^{|\gamma|} \quad \text { for }|\gamma| \leqq[n / 2]+1 .
$$

Then $m \in \mathcal{M}\left(C^{s}\left(\mathbb{R}^{n}\right), C^{s+\theta}\left(\mathbb{R}^{n}\right)\right)$, and

$$
\|m\|_{\mathcal{M}\left(C^{s}\left(\mathbb{R}^{n}\right), C^{s+\theta}\left(\mathbb{R}^{n}\right)\right)} \leq C A^{\frac{n}{2}},
$$

where the constant $C$ depends only on $a, s, \theta$ and $n$.

2.6. Faá di Bruno's formula. Let us also recall Faá di Bruno's formula for later use. In the following lemma, we use bold symbols to distinguish multi-index integers from integers.

Lemma 2.14. [3, 2.10. Corollary] Assume that $x \in \mathbb{R}^{n}$ and $y \in \mathbb{R}$. Let $|\gamma| \geq 1$, and $h\left(x_{1}, x_{2}, \cdots, x_{n}\right)=f\left(g\left(x_{1}, x_{2}, \cdots, x_{n}\right)\right)$ with $g \in C^{|\gamma|}\left(\mathbb{R}^{n}\right)$ and $f \in C^{|\gamma|}(\mathbb{R})$, let $y=g(x)$. There holds:

$$
D^{\gamma} h=\sum_{r=1}^{|\gamma|} \frac{d^{r} f}{d y^{r}} \sum_{p(\boldsymbol{\gamma}, r)}(\gamma !) \prod_{j=1}^{|\gamma|} \frac{\left[D^{\ell_{j}} g\right]^{k_{j}}}{\left(k_{j} !\right)\left(\boldsymbol{\ell}_{j} !\right)^{k_{j}}}
$$

where

$$
\begin{gathered}
p(\boldsymbol{\gamma}, r)=\left\{\left(k_{1}, \ldots, k_{|\gamma|} ; \boldsymbol{\ell}_{1}, \ldots, \boldsymbol{\ell}_{|\gamma|}\right): \text { for some } 1 \leq s \leq|\gamma|,\right. \\
k_{i}=0 \text { and } \boldsymbol{\ell}_{i}=\mathbf{0} \text { for } 1 \leq i \leq|\gamma|-s ; k_{i}>0 \text { for }|\gamma|-s+1 \leq i \leq|\gamma| ; \\
\text { and } \mathbf{0} \prec \boldsymbol{\ell}_{n-s+1} \prec \cdots \prec \boldsymbol{\ell}_{n} \text { are such that } \\
\left.\sum_{i=1}^{n} k_{i}=r, \sum_{i=1}^{n} k_{i} \boldsymbol{\ell}_{i}=\gamma\right\} .
\end{gathered}
$$

2.7. Classical solutions and mild solutions. In this subsection, we give the definitions of classical and mild solutions for (1.1).

Definition 2.15 (Classical solution). Suppose $u_{0}, u_{1} \in C\left(\mathbb{R}^{n}\right)$ and $f \in C([0, \infty) \times$ $\left.\mathbb{R}^{n}\right)$. Then a function $u(t, x) \in C\left([0, \infty) \times \mathbb{R}^{n}\right)$ is called a classical solution of the Cauchy problem (1.1) if

(1) $\Delta u(t, x)$ defines a continuous function of $x$ for every $t>0$,

(2) for every $x \in \mathbb{R}^{n}$, the fractional integral $I_{t}^{2-\alpha} u^{\prime}$, where $u^{\prime}=\frac{\partial u}{\partial t}$,

$$
I_{t}^{2-\alpha} u^{\prime}(t, x)=\frac{1}{\Gamma(2-\alpha)} \int_{0}^{t}(t-\tau)^{1-\alpha} \frac{\partial u}{\partial t}(\tau, x) \mathrm{d} \tau,
$$

is continuously differentiable with respect to $t>0$, and 
(3) $u(t, x)$ satisfies the equation (1.1) for every $(t, x) \in(0, \infty) \times \mathbb{R}^{n}$ and the initial conditions in (1.1) for every $x \in \mathbb{R}^{n}$.

Recall that $E_{\alpha, \beta}(z)$ is the Mittag-Leffler function which is given in Definition 2.4. Define

$$
\begin{aligned}
\widehat{S}_{\alpha}(t, \xi) & =E_{\alpha, 1}\left(i^{-\alpha} t^{\alpha}|\xi|^{2}\right), \\
\widehat{Q}_{\alpha}(t, \xi) & =t E_{\alpha, 2}\left(i^{-\alpha} t^{\alpha}|\xi|^{2}\right), \\
\widehat{P}_{\alpha}(t, \xi) & =i^{-\alpha} t^{\alpha-1} E_{\alpha, \alpha}\left(i^{-\alpha} t^{\alpha}|\xi|^{2}\right),
\end{aligned}
$$

and $S_{\alpha}(t, x), Q_{\alpha}(t, x), P_{\alpha}(t, x)$ be the inverse Fourier transform of $\widehat{S}_{\alpha}(t, \xi), \widehat{Q}_{\alpha}(t, \xi), \widehat{P}_{\alpha}(t, \xi)$ respectively. We write $S_{\alpha}(x)=S_{\alpha}(1, x), Q_{\alpha}(x)=Q_{\alpha}(1, x)$, and $P_{\alpha}(x)=P_{\alpha}(1, x)$ for short.

When the initial data is sufficiently regular, we can use the Laplace and Fourier transforms to write the solution as the sum of three terms,

$$
\begin{aligned}
u(t, x) & =\int_{\mathbb{R}^{n}} S_{\alpha}(t, x-y) u_{0}(y) \mathrm{d} y+\int_{\mathbb{R}^{n}} S_{\alpha}(t, x-y) u_{1}(y) \mathrm{d} y+\int_{0}^{t} \int_{\mathbb{R}^{n}} P_{\alpha}(t-\tau, x-y) f(\tau, y) \mathrm{d} y \mathrm{~d} \tau \\
& \left.=\mathcal{F}^{-1}\left(\widehat{S}_{\alpha}(t, \xi) \widehat{u}_{0}(\xi)\right)+\mathcal{F}^{-1}\left(\widehat{Q}_{\alpha}(t, \xi) \widehat{u}_{1}(\xi)\right)+\mathcal{F}^{-1}\left(\int_{0}^{t} \widehat{P}_{\alpha}(t-\tau, \xi)\right) \widehat{f}(\tau, \xi) \mathrm{d} \tau\right) \\
& =: u_{0}(t, x)+u_{1}(t, x)+u_{f}(t, x) .
\end{aligned}
$$

Definition 2.16 (Mild solution). Let $u_{0}, u_{1}$ and $f$ be measurable functions on $\mathbb{R}^{n}$ and $[0, \infty) \times \mathbb{R}^{n}$, respectively. Then the function $u$ defined by (2.13) is called the mild solution of the Cauchy problem (1.1) whenever the integrals in (2.13) are well defined.

Remark 2.17. If $u_{0}, u_{1} \in \mathcal{S}$ and $f \in \mathcal{S}$ for each $t>0$, then the mild solution is a classical solution.

Using (2.6), as $t|\xi|^{\frac{2}{\alpha}} \rightarrow \infty$, we have the following results:

$$
\begin{aligned}
& \widehat{S}_{\alpha}(t, \xi)=\frac{1}{\alpha} e^{-i t|\xi|^{\frac{2}{\alpha}}}-\sum_{k=1}^{m} \frac{i^{\alpha k} t^{-\alpha k}}{\Gamma(1-\alpha k)|\xi|^{2 k}}+o\left(\frac{t^{-\alpha m}}{|\xi|^{2 m}}\right) \\
& \widehat{Q}_{\alpha}(t, \xi)=\frac{1}{\alpha} i|\xi|^{-\frac{2}{\alpha}} e^{-i t|\xi|^{\frac{2}{\alpha}}}-\sum_{k=1}^{m} \frac{i^{\alpha k} t^{1-\alpha k}}{\Gamma(2-\alpha k)|\xi|^{2 k}}+o\left(\frac{t^{1-\alpha m}}{|\xi|^{2 m}}\right), \text { and } \\
& \widehat{P}_{\alpha}(t, \xi)=\frac{1}{\alpha} i^{-1}|\xi|^{-\frac{2(\alpha-1)}{\alpha}} e^{-i t|\xi|^{\frac{2}{\alpha}}}-\sum_{k=2}^{m} \frac{i^{\alpha k-\alpha} t^{\alpha-1-\alpha k}}{\Gamma(\alpha-\alpha k)|\xi|^{2 k}}+o\left(\frac{t^{\alpha-1-\alpha m}}{|\xi|^{2 m}}\right) .
\end{aligned}
$$

It is obvious that $\widehat{S}_{\alpha}(t, \xi), \widehat{Q}_{\alpha}(t, \xi), \widehat{P}_{\alpha}(t, \xi)$ are oscillating as $|\xi| \rightarrow \infty$ for any fixed $t>0$.

Remark 2.18. It is easy to check that $\widehat{S}_{\alpha}(t, \xi)=D_{t}^{1} \widehat{Q}_{\alpha}(t, \xi)$ and $\widehat{P}_{\alpha}(t, \xi)=$ $D_{t}^{2-\alpha} \widehat{Q}_{\alpha}(t, \xi)$.

\section{Asymptotic PRoperties of the KERnels}

In this section, we give the asymptotics for the oscillatory kernels and its Bessel potentials for $t=1$. In addition, we derive some upper bounds for the the Bessel potentials of the kernels for any $t>0$. 
First, we consider the case when $t=1$. Recall that, for any $\theta \geq 0$,

$$
\begin{aligned}
& J^{\theta} S_{\alpha}(x)=\frac{1}{(2 \pi)^{n / 2}} \int_{\mathbb{R}^{n}} e^{i x \cdot \xi}\left(1+|\xi|^{2}\right)^{-\theta / 2} E_{\alpha, 1}\left(i^{-\alpha}|\xi|^{2}\right) \mathrm{d} \xi, \\
& J^{\theta} Q_{\alpha}(x)=\frac{1}{(2 \pi)^{n / 2}} \int_{\mathbb{R}^{n}} e^{i x \cdot \xi}\left(1+|\xi|^{2}\right)^{-\theta / 2} E_{\alpha, 2}\left(i^{-\alpha}|\xi|^{2}\right) \mathrm{d} \xi, \text { and } \\
& J^{\theta} P_{\alpha}(x)=\frac{1}{(2 \pi)^{n / 2}} \int_{\mathbb{R}^{n}} e^{i x \cdot \xi}\left(1+|\xi|^{2}\right)^{-\theta / 2} E_{\alpha, \alpha}\left(i^{-\alpha}|\xi|^{2}\right) \mathrm{d} \xi
\end{aligned}
$$

Then we have the following lemma.

Lemma 3.1. For any $\theta \geq 0$. Then $J^{\theta} S_{\alpha}(x), J^{\theta} Q_{\alpha}(x)$ and $J^{\theta} P_{\alpha}(x)$ are all smooth functions throughout $\mathbb{R}^{n} \backslash\{0\}$ with the following asymptotic behavior as $|x| \rightarrow \infty$,

$$
\begin{aligned}
& J^{\theta} S_{\alpha}(x)=A|x|^{\frac{n \alpha-n-\theta \alpha}{2-\alpha}} e^{-i B|x|^{\frac{2}{\alpha-2}}}+o\left(|x|^{\frac{n \alpha-n-\theta \alpha}{2-\alpha}}\right), \\
& J^{\theta} Q_{\alpha}(x)=A|x|^{\frac{n \alpha-n-2-\alpha \theta}{2-\alpha}} e^{-i B|x|^{\frac{2}{\alpha-2}}}+o\left(|x|^{\frac{n \alpha-n-2-\alpha \theta}{2-\alpha}}\right), \text { and } \\
& J^{\theta} P_{\alpha}(x)=A|x|^{\frac{n \alpha+2-n-2 \alpha-\alpha \theta}{2-\alpha}} e^{-i B|x|^{\frac{2}{\alpha-2}}}+o\left(|x|^{\frac{n \alpha+2-n-2 \alpha-\alpha \theta}{2-\alpha}}\right),
\end{aligned}
$$

where $A$ and $B$ are some constants (which may differ from line to line) which only depend on $\alpha, \theta$ and $n$. They have the following asymptotic behavior as $|x| \rightarrow 0$,

$$
\left|J^{\theta} S_{\alpha}(x)\right|,\left|J^{\theta} Q_{\alpha}(x)\right| \sim \begin{cases}C, & \text { when } \theta>n-2, \\ C \log \frac{1}{|x|}, & \text { when } \theta=n-2, \\ C|x|^{2+\theta-n}+o\left(|x|^{2+\theta-n}\right), & \text { when } \theta<n-2,\end{cases}
$$

and

$$
\left|J^{\theta} P_{\alpha}(x)\right| \sim \begin{cases}C, & \text { when } \theta>n-4, \\ C \log \frac{1}{|x|}, & \text { when } \theta=n-4 \\ C|x|^{4+\theta-n}+o\left(|x|^{4+\theta-n}\right), & \text { when } \theta<n-4 .\end{cases}
$$

In particular, define the constants

$$
\theta_{S_{\alpha}}=\frac{n}{\alpha}, \theta_{Q_{\alpha}}=\frac{n}{\alpha}-\frac{2}{\alpha} \text { and } \theta_{P_{\alpha}}=\frac{n}{\alpha}+\frac{2}{\alpha}-2 .
$$

Then

(1) if $\theta>\theta_{S_{\alpha}}$, we have $J^{\theta} S_{\alpha}(x) \in L^{1}\left(\mathbb{R}^{n}\right)$;

(2) if $\theta>\theta_{Q_{\alpha}}$, we have $J^{\theta} Q_{\alpha}(x) \in L^{1}\left(\mathbb{R}^{n}\right)$; and

(3) if $\theta>\theta_{P_{\alpha}}$, we have $J^{\theta} P_{\alpha}(x) \in L^{1}\left(\mathbb{R}^{n}\right)$.

Proof. Since the proofs for the three kernels are similar, we only give the details for $S_{\alpha}(x)$.

Denote by $\Phi(\xi)$ a fixed smooth function that is supported in the unit ball centered at the origin. Recall that $\widehat{J}^{\theta} S_{\alpha}(\xi)=\left(1+|\xi|^{2}\right)^{-\theta / 2} E_{\alpha, 1}\left(i^{-\alpha}|\xi|^{2}\right)$ is locally integrable, thus it belongs to $\mathcal{S}^{\prime}\left(\mathbb{R}^{n}\right)$. Then we can write $J^{\theta} S_{\alpha}(x)$ as follows (interpreted in 
the sense of distributions),

$$
\begin{aligned}
J^{\theta} S_{\alpha}(x)= & \frac{1}{(2 \pi)^{n / 2}} \int_{\mathbb{R}^{n}} e^{i x \cdot \xi}\left(1+|\xi|^{2}\right)^{-\theta / 2} E_{\alpha, 1}\left(i^{-\alpha}|\xi|^{2}\right) \mathrm{d} \xi \\
= & \frac{1}{(2 \pi)^{n / 2}} \int_{\mathbb{R}^{n}} e^{i x \cdot \xi}\left(1+|\xi|^{2}\right)^{-\theta / 2} E_{\alpha, 1}\left(i^{-\alpha}|\xi|^{2}\right) \Phi(\xi) \mathrm{d} \xi \\
& +\frac{1}{(2 \pi)^{n / 2}} \int_{\mathbb{R}^{n}} e^{i x \cdot \xi}\left(1+|\xi|^{2}\right)^{-\theta / 2} E_{\alpha, 1}\left(i^{-\alpha}|\xi|^{2}\right)(1-\Phi(\xi)) \mathrm{d} \xi \\
= & : S_{\alpha, \theta, 1}(x)+S_{\alpha, \theta, 2}(x) .
\end{aligned}
$$

Since $\left(1+|\xi|^{2}\right)^{-\theta / 2} E_{\alpha, 1}\left(i^{-\alpha}|\xi|^{2}\right) \Phi(\xi) \in C_{c}^{\infty}\left(\mathbb{R}^{n}\right)$, we have that $S_{\alpha, \theta, 1}(x)$ is a Schwartz function. In particular, for any integer $N \geq 1$,

$$
\left|S_{\alpha, \theta, 1}(x)\right| \lesssim\left\{\begin{array}{lll}
1 & \text { as } & |x| \rightarrow 0 \\
|x|^{-N}, & \text { as } & |x| \rightarrow \infty
\end{array}\right.
$$

For the second term, we rewrite it as

$$
\begin{aligned}
S_{\alpha, \theta, 2}(x) & =\frac{1}{(2 \pi)^{n / 2}} \int_{\mathbb{R}^{n}} e^{i x \cdot \xi}\left(1+|\xi|^{2}\right)^{-\theta / 2} E_{\alpha, 1}\left(i^{-\alpha}|\xi|^{2}\right)(1-\Phi(\xi)) \mathrm{d} \xi \\
& =\frac{1}{(2 \pi)^{n / 2}} \int_{\mathbb{R}^{n}} e^{i x \cdot \xi}\left(1+|\xi|^{2}\right)^{-\theta / 2} \frac{1}{\alpha} e^{-i|\xi|^{\frac{2}{\alpha}}}(1-\Phi(\xi)) \mathrm{d} \xi \\
& +\frac{1}{(2 \pi)^{n / 2}} \int_{\mathbb{R}^{n}} e^{i x \cdot \xi}\left(1+|\xi|^{2}\right)^{-\theta / 2}\left(E_{\alpha, 1}\left(i^{-\alpha}|\xi|^{2}\right)-\frac{1}{\alpha} e^{-i|\xi|^{\frac{2}{\alpha}}}\right)(1-\Phi(\xi)) \mathrm{d} \xi \\
& =: S_{\alpha, \theta, 21}(x)+S_{\alpha, \theta, 22}(x) .
\end{aligned}
$$

By Corollary 2.12, $S_{\alpha, \theta, 2}(x)$ is a smooth function throughout $\mathbb{R}^{n}$, and

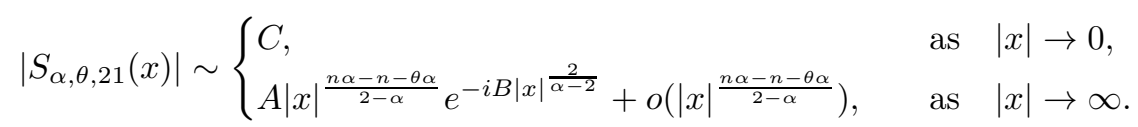

Now we deal with $S_{\alpha, \theta, 22}(x)$. Define $r_{\alpha, \theta}(\xi)$ as the following term which appears in the integrand of $S_{\alpha, \theta, 22}(x)$,

$$
r_{\alpha, \theta}(\xi):=\left(1+|\xi|^{2}\right)^{-\theta / 2}\left(E_{\alpha, 1}\left(i^{-\alpha}|\xi|^{2}\right)-\frac{1}{\alpha} e^{-i|\xi|^{\frac{2}{\alpha}}}\right)(1-\Phi(\xi)) .
$$

Employing the asymptotic formula (2.6), we have for any integer $M \geq 1$,

$$
\left.r_{\alpha, \theta}(\xi)=\left(\sum_{k=1}^{M} \frac{C_{k}}{|\xi|^{2 k+\theta}}+o\left(\frac{1}{|\xi|^{2 k+\theta}}\right)\right)(1-\Phi(\xi))\right) .
$$

Using integration by parts, it is easy to see for any integer $N \geq 1$

$$
\left|S_{\alpha, \theta, 22}(x)\right| \lesssim|x|^{-N}, \quad \text { as } \quad x \rightarrow \infty .
$$

It remains to consider the case when $|x| \rightarrow 0$. Using similar estimates for Lemma 3.1 in [32, we have the following expansion for $S_{\alpha, \theta, 22}(x)$,

(1) If $n>\theta+2$, for some positive integer $M$,

$$
S_{\alpha, \theta, 22}(x)=\sum_{k=1}^{M} C_{k}|x|^{-n+2 k+\theta}+W(x), \quad \text { as } \quad|x| \rightarrow 0,
$$

where $W \in L^{\infty}\left(\mathbb{R}^{n}\right)$. 
(2) If $n=\theta+2$, for some positive integer $M$,

$$
S_{\alpha, \theta, 22}(x)=\sum_{k=1}^{M} C_{k}|x|^{-n+2 k+\theta}+C W_{1}(x)+W(x),
$$

where $W \in L^{\infty}\left(\mathbb{R}^{n}\right)$, and $W_{1}(x) \sim \ln |x|$, as $|x| \rightarrow 0$.

(3) If $n<\theta+2$, then $r_{\alpha, \theta}(\xi)$ is integrable. Thus $S_{\alpha, \theta, 22}(x)$ is bounded as $|x| \rightarrow 0$.

In particular, we have

$$
\left|S_{\alpha, \theta, 22}(x)\right| \sim \begin{cases}C, & \text { when } \quad n<2+\theta, \\ \log \frac{1}{|x|}, & \text { when } n=2+\theta, \quad \text { as } \quad|x| \rightarrow 0 . \\ |x|^{2+\theta-n}, & \text { when } n>2+\theta,\end{cases}
$$

Combining (3.7), (3.8), (3.9) and (3.10), gives the asymptotic behavior for $J^{\theta} S_{\alpha}(x)$. Notice that, when $\theta>\frac{n}{\alpha}$, we have $\frac{n \alpha-n-\theta \alpha}{2-\alpha}<-n$, which implies $J^{\theta} S_{\alpha} \in L^{1}\left(\mathbb{R}^{n}\right)$.

As for $Q_{\alpha}(x)$ and $P_{\alpha}(x)$, we can derive the desired results using the same method.

Now we compare the properties of our kernels with some known results. Here we take $\theta=0$ and $S_{\alpha}(x)$ to illustrate our ideas. From Lemma 3.1. $S_{\alpha}(x)$ has the following asymptotic behavior as $|x| \rightarrow \infty$,

$$
S_{\alpha}(x)=A|x|^{\frac{n \alpha-n}{2-\alpha}} e^{-i B|x|^{\frac{2}{\alpha-2}}}+o\left(|x|^{\frac{n \alpha-n}{2-\alpha}}\right),
$$

and have the following asymptotic behavior as $|x| \rightarrow 0$,

$$
\left|S_{\alpha}(x)\right| \sim\left\{\begin{array}{lr}
C, & \text { when } n<2, \\
C \log \frac{1}{|x|}, & \text { when } n=2 \\
C|x|^{2-n}+o\left(|x|^{2-n}\right), & \text { when } n>2 .
\end{array}\right.
$$

Let us denote $S_{\alpha}^{\mathrm{h}}(x)=\mathcal{F}^{-1}\left(E_{\alpha, 1}\left(-|\xi|^{2}\right)\right)$, which is the fundamental solution for the fractional diffusion-wave equation (1.2). According to the Theorem 2.1 and Theorem 2.2 in [15], $S_{\alpha}^{\mathrm{h}}(x)$ has the following asymptotic behavior as $|x| \rightarrow \infty$,

$$
\left|S_{\alpha}^{\mathrm{h}}(x)\right| \lesssim|x|^{-n} e^{-c|x|^{\frac{2}{2-\alpha}}}
$$

where $c>0$ is a constant, and has the following asymptotic behavior as $|x| \rightarrow 0$,



Remark 3.2. Suppose that $1<\alpha<2$. As $|x| \rightarrow 0$, according to (3.12) and (3.14), $S_{\alpha}^{\mathrm{h}}(x)$ and $S_{\alpha}(x)$ have the same behavior and they are both in $L_{\text {loc }}^{1}\left(\mathbb{R}^{n}\right)$. As $|x| \rightarrow \infty$, due to (3.13), $S_{\alpha}^{\mathrm{h}}(x)$ has exponential decay. In contrast, by (3.11), the main term for $S_{\alpha}(x)$ is an increasing polynomial multiplied by an oscillatory term, which causes the solution for (1.1) to lose regularity.

Remark 3.3. We can also compare $S_{\alpha}(x)$ with $K_{0, \frac{2}{\alpha}}^{-}(x)$. It is easy to see that they have the same asymptotic behavior as $|x| \rightarrow \infty$; However, they behaves differently as $|x| \rightarrow 0$, since the former one may has singularity at $|x|=0$ when the dimension 
is large. This difference is caused by the reminder term $r(\xi)$ of $E_{\alpha, 1}\left(i^{\alpha}|\xi|^{2}\right)$ as $|\xi| \rightarrow \infty$.

In the next lemma, we give some upper bounds for the Bessel potentials of the kernels for any arbitrary $t>0$. Since $\left(1+|\xi|^{2}\right)^{-\theta / 2}$ is not homogeneous, the following results cannot be obtained by scaling. Recall that $\theta_{S_{\alpha}}, \theta_{Q_{\alpha}}$ and $\theta_{P_{\alpha}}$ are constants defined in (3.6).

Lemma 3.4. For the Bessel potentials of the kernels $S_{\alpha}(t, x), Q_{\alpha}(t, x)$ and $P_{\alpha}(t, x)$ with arbitrary $t>0$, we have the following upper bounds:

(1) (a) If $\theta>\theta_{S_{\alpha}}$ and $0<t<1$, then

$$
\left|J^{\theta} S_{\alpha}(t, x)\right| \leq \begin{cases}C \max \left\{1,|x|^{\theta-n}\right\}, & |x| \leq t^{\frac{\alpha}{2}}, \\ C \max \left\{1, t^{\frac{\alpha}{2}(\theta-n)}\right\}, & t^{\frac{\alpha}{2}} \leq|x| \leq 1, \\ C|x|^{-n-\sigma}, & |x| \geq 1,\end{cases}
$$

where $\sigma=\frac{\theta \alpha-n}{2-\alpha}>0$. In particular, $\int_{R^{n} \backslash B(0,1)}\left|J^{\theta} S_{\alpha}(t, x)\right| \mathrm{d} x$ is bounded uniformly in $0<t<1$, where $B(0,1)$ is the unit ball of $\mathbb{R}^{n}$ centered at the origin.

(b) If $\theta>\theta_{S_{\alpha}}$ and $t \geq 1$, then

$$
\left|J^{\theta} S_{\alpha}(t, x)\right| \leq \begin{cases}C \max \left\{1,|x|^{\theta-n}\right\}, & |x| \leq t^{\frac{\alpha}{2}} \\ C t^{\frac{\alpha}{2}(\theta+\sigma)}|x|^{-n-\sigma}, & |x| \geq t^{\frac{\alpha}{2}}\end{cases}
$$

where $\sigma=\frac{\theta \alpha-n}{2-\alpha}>0$.

(2) (a) For $\theta>\theta_{Q_{\alpha}}$ and $0<t<1$, then

$$
\left|J^{\theta} Q_{\alpha}(t, x)\right| \leq \begin{cases}C \max \left\{1,|x|^{\theta-n+\frac{2}{\alpha}}\right\}, & |x| \leq t^{\frac{\alpha}{2}}, \\ C \max \left\{1, t^{\frac{\alpha}{2}\left(\theta-n+\frac{2}{\alpha}\right)}\right\}, & t^{\frac{\alpha}{2}} \leq|x| \leq 1, \\ C|x|^{-n-\sigma}, & |x| \geq 1,\end{cases}
$$

where $\sigma=\frac{\theta \alpha+2-n}{2-\alpha}>0$. In particular, $\int_{R^{n} \backslash B(0,1)}\left|J^{\theta} Q_{\alpha}(t, x)\right| \mathrm{d} x$ is bounded uniformly in $0<t<1$.

(b) For $\theta>\theta_{Q_{\alpha}}$ and $t \geq 1$, then

$$
\left|J^{\theta} Q_{\alpha}(t, x)\right| \leq \begin{cases}C \max \left\{1,|x|^{\theta-n+\frac{2}{\alpha}}\right\}, & |x| \leq t^{\frac{\alpha}{2}}, \\ C t^{\frac{\alpha}{2}(\theta+\sigma)}|x|^{-n-\sigma}, & |x| \geq t^{\frac{\alpha}{2}},\end{cases}
$$

where $\sigma=\frac{\theta \alpha+2-n}{2-\alpha}>0$.

(3) (a) For $\theta>\theta_{P_{\alpha}}$ and $0<t<1$, then

$$
\left|J^{\theta} P_{\alpha}(t, x)\right| \leq \begin{cases}C \max \left\{1,|x|^{\theta-n+2-\frac{2}{\alpha}}\right\}, & |x| \leq t^{\frac{\alpha}{2}} \\ C \max \left\{1, t^{\frac{\alpha}{2}\left(\theta-n+2-\frac{2}{\alpha}\right)}\right\}, & t^{\frac{\alpha}{2}} \leq|x| \leq 1, \\ C|x|^{-n-\sigma}, & |x| \geq 1,\end{cases}
$$

where $\sigma=\frac{\theta \alpha+2 \alpha-2-n}{2-\alpha}>0$. In particular, $\int_{R^{n} \backslash B(0,1)}\left|J^{\theta} P_{\alpha}(t, x)\right| \mathrm{d} x$ is bounded uniformly in $0<t<1$.

(b) For $\theta>\theta_{P_{\alpha}}$ and $t \geq 1$, then

$$
\left|J^{\theta} P_{\alpha}(t, x)\right| \leq \begin{cases}C \max \left\{1,|x|^{\theta-n+2-\frac{2}{\alpha}}\right\}, & |x| \leq t^{\frac{\alpha}{2}}, \\ C t^{\frac{\alpha}{2}(\theta+\sigma)}|x|^{-n-\sigma}, & |x| \geq t^{\frac{\alpha}{2}},\end{cases}
$$

where $\sigma=\frac{\theta \alpha+2 \alpha-2-n}{2-\alpha}>0$. 
Proof. We only prove the estimates for $J^{\theta} S_{\alpha}(t, x)$, because the estimates for the other two terms are similarly derived. Performing a change of variables, we can write

$$
\begin{aligned}
J^{\theta} S_{\alpha}(t, x) & =\frac{1}{(2 \pi)^{n / 2}} \int_{\mathbb{R}^{n}} e^{i x \cdot \xi}\left(1+|\xi|^{2}\right)^{-\frac{\theta}{2}} E_{\alpha, 1}\left(i^{-\alpha} t^{\alpha}|\xi|^{2}\right) \mathrm{d} \xi \\
& =\frac{1}{(2 \pi)^{n / 2}} t^{-\alpha n / 2} \int_{\mathbb{R}^{n}} e^{i x t^{-\frac{\alpha}{2}} \cdot \xi}\left(1+t^{-\alpha}|\xi|^{2}\right)^{-\frac{\theta}{2}} E_{\alpha, 1}\left(i^{-\alpha}|\xi|^{2}\right) \mathrm{d} \xi \\
& =\frac{1}{(2 \pi)^{n / 2}} t^{-\alpha n / 2} \int_{\mathbb{R}^{n}} e^{i x t^{-\frac{\alpha}{2}} \cdot \xi}\left(1+t^{-\alpha}|\xi|^{2}\right)^{-\frac{\theta}{2}} E_{\alpha, 1}\left(i^{-\alpha}|\xi|^{2}\right) \Phi(\xi) \mathrm{d} \xi \\
& +\frac{1}{(2 \pi)^{n / 2}} t^{-\alpha n / 2} \int_{\mathbb{R}^{n}} e^{i x t^{-\frac{\alpha}{2}} \cdot \xi}\left(1+t^{-\alpha}|\xi|^{2}\right)^{-\frac{\theta}{2}} E_{\alpha, 1}\left(i^{-\alpha}|\xi|^{2}\right)(1-\Phi(\xi)) \mathrm{d} \xi \\
& :=I_{1}(t, x)+I_{2}(t, x) .
\end{aligned}
$$

Case $(a)$ : when $0<t<1$.

Step 1: First, we claim that, for any integral $N$, the following estimate is valid,

$$
\left|I_{1}(t, x)\right| \lesssim \min \left\{\max \left\{1, t^{\alpha / 2(\theta-n)}\right\}, \frac{t^{\alpha / 2(\theta+N-n)}+1}{|x|^{N}}\right\}
$$

Then, we have for $N$ large enough,

$$
\left|I_{1}(t, x)\right| \lesssim \begin{cases}\max \left\{1,|x|^{\theta-n}\right\}, & |x| \leq t^{\alpha / 2} \\ \max \left\{1, t^{\frac{\alpha}{2}(\theta-n)}\right\} & t^{\alpha / 2} \leq|x| \leq 1 \\ \frac{1}{|x|^{N}}, & |x| \geq 1\end{cases}
$$

The claim (3.21) is valid, because on the one hand, by spliting the integral into two parts, i.e. $|\xi| \leq t^{\frac{\alpha}{2}}$ and $|\xi| \geq t^{\frac{\alpha}{2}}$, we have the following trivial bound,

$$
\begin{aligned}
\left|I_{1}(t, x)\right| & \lesssim t^{-\frac{\alpha}{2} n} \int_{\mathbb{R}^{n}}\left(1+t^{-\alpha}|\xi|^{2}\right)^{-\frac{\theta}{2}} \Phi(\xi) \mathrm{d} \xi \\
& \lesssim t^{-\frac{\alpha}{2} n} \int_{|\xi| \leq t^{\frac{\alpha}{2}}} \Phi(\xi) \mathrm{d} \xi+t^{-\frac{\alpha}{2} n} \int_{t^{\frac{\alpha}{2}} \leq|\xi| \leq 1} t^{\frac{\alpha}{2} \theta}|\xi|^{-\theta} \Phi(\xi) \mathrm{d} \xi \\
& \lesssim t^{-\frac{\alpha}{2} n} t^{-\frac{\alpha}{2} n}+\left(1+t^{\frac{\alpha}{2}(\theta-n)}\right) \\
& \lesssim \max \left\{1, t^{\frac{\alpha}{2}(\theta-n)}\right\} .
\end{aligned}
$$

On the other hand, define

$$
\mathcal{D} u=-i \sum_{j=1}^{n} \frac{x_{j} t^{-\alpha / 2}}{\left|x t^{-\alpha / 2}\right|} \partial_{j} u
$$

then it is easy to see that its adjoint operator is ${ }^{T} \mathcal{D}=-\mathcal{D}$ and

$$
\mathcal{D} e^{i x t^{-\frac{\alpha}{2}} \cdot \xi}=\left|x t^{-\alpha / 2}\right| e^{i x t^{-\frac{\alpha}{2}} \cdot \xi}
$$


Hence by integrating by parts $N$ times, we have

$$
\begin{aligned}
I_{1}(t, x) & =\frac{1}{(2 \pi)^{n / 2}} t^{-\alpha n / 2} \int_{\mathbb{R}^{n}} \frac{1}{\left|i x t^{-\alpha / 2}\right|^{N}} \mathcal{D}^{N} e^{i x t^{-\frac{\alpha}{2}} \cdot \xi}\left[\left(1+t^{-\alpha}|\xi|^{2}\right)^{-\frac{\theta}{2}} E_{\alpha, 1}\left(i^{-\alpha}|\xi|^{2}\right) \Phi(\xi)\right] \mathrm{d} \xi \\
& =\frac{1}{(2 \pi)^{n / 2}} t^{-\alpha n / 2} \frac{1}{\left|-i x t^{-\alpha / 2}\right|^{N}} \int_{\mathbb{R}^{n}} e^{i x t^{-\frac{\alpha}{2}} \cdot \xi} \mathcal{D}^{N}\left[\left(1+t^{-\alpha}|\xi|^{2}\right)^{-\frac{\theta}{2}} E_{\alpha, 1}\left(i^{-\alpha}|\xi|^{2}\right) \Phi(\xi)\right] \mathrm{d} \xi \\
& =\frac{1}{(2 \pi)^{n / 2}} t^{-\alpha n / 2} \frac{1}{\left|-i x t^{-\alpha / 2}\right|^{N}} \int_{|\xi| \leq t^{\alpha / 2}} e^{i x t^{-\frac{\alpha}{2}} \cdot \xi} \mathcal{D}^{N}\left[\left(1+t^{-\alpha}|\xi|^{2}\right)^{-\frac{\theta}{2}} E_{\alpha, 1}\left(i^{-\alpha}|\xi|^{2}\right) \Phi(\xi)\right] \mathrm{d} \xi \\
& +\frac{1}{(2 \pi)^{n / 2}} t^{-\alpha n / 2} \frac{1}{\left|-i x t^{-\alpha / 2}\right|^{N}} \int_{t^{\frac{\alpha}{2}} \leq|\xi| \leq 1} e^{i x t^{-\frac{\alpha}{2}} \cdot \xi} \mathcal{D}^{N}\left[\left(1+t^{-\alpha}|\xi|^{2}\right)^{-\frac{\theta}{2}} E_{\alpha, 1}\left(i^{-\alpha}|\xi|^{2}\right) \Phi(\xi)\right] \mathrm{d} \xi \\
& =: I_{11}(t, x)+I_{12}(t, x) .
\end{aligned}
$$

For $I_{11}(t, x)$, since $t^{-\alpha}|\xi|^{2} \leq 1$, direct calculation yields

$$
\left|\mathcal{D}^{N}\left[\left(1+t^{-\alpha}|\xi|^{2}\right)^{-\frac{\theta}{2}} E_{\alpha, 1}\left(i^{-\alpha}|\xi|^{2}\right)\right]\right| \lesssim t^{-\frac{\alpha}{2} N}
$$

Thus,

$$
\left|I_{11}(t, x)\right| \lesssim \frac{t^{-\alpha n / 2}}{|x|^{N}} \int_{|\xi| \leq t^{\alpha / 2}} \mathrm{~d} \xi \lesssim \frac{1}{|x|^{N}}
$$

For $I_{22}(t, x)$, since $t^{-\alpha}|\xi|^{2} \geq 1$, we have $\mathcal{D}^{N}\left[\left(1+t^{-\alpha}|\xi|^{2}\right)^{-\frac{\theta}{2}}\right] \sim t^{\frac{\theta \alpha}{2}}|\xi|^{\theta-N}$. Thus,

$$
\begin{aligned}
\left|I_{12}(t, x)\right| & \lesssim \frac{t^{-\alpha n / 2}}{\left|x t^{-\alpha / 2}\right|^{N}} \int_{t^{\frac{\alpha}{2}} \leq|\xi| \leq 1} t^{\frac{\alpha}{2} \theta}|\xi|^{-\theta-N} \mathrm{~d} \xi \\
& \lesssim \frac{t^{\alpha / 2(\theta+N-n)}}{|x|^{N}}\left(1+t^{\alpha / 2(n-\theta-N)}\right) \lesssim \frac{t^{\alpha / 2(\theta+N-n)}+1}{|x|^{N}} .
\end{aligned}
$$

Combining (3.23), (3.24), and (3.25), we obtain

$$
\left|I_{1}(t, x)\right| \lesssim \min \left\{\max \left\{1, t^{\alpha / 2(\theta-n)}\right\}, \frac{t^{\alpha / 2(\theta+N-n)}+1}{|x|^{N}}\right\} .
$$

Step 2: Next we deal with $I_{2}(t, x)$. In order to use the scaling property, let us introduce $\tilde{I}_{2}(t, x)$, defined as

$$
\tilde{I}_{2}(t, x):=\frac{1}{(2 \pi)^{n / 2}} t^{-\alpha n / 2} \int_{\mathbb{R}^{n}} e^{i x t^{-\alpha / 2} \cdot \xi} t^{\frac{\alpha \theta}{2}}|\xi|^{-\theta} E_{\alpha, 1}\left(i^{-\alpha}|\xi|^{2}\right)(1-\Phi(\xi)) \mathrm{d} \xi .
$$

In this case $t^{-\alpha / 2}|\xi| \geq 1$, thus $I_{2}(t, x)$ and $\tilde{I}_{2}(t, x)$ has the same asymptotic as $\left|x t^{-\frac{\alpha}{2}}\right| \rightarrow 0$ or $\left|x t^{-\frac{\alpha}{2}}\right| \rightarrow \infty$. By the estimates of (3.8), (3.9) and (3.10), when $\theta>\frac{n}{\alpha}$, we have

$$
\left|I_{2}(t, x)\right| \sim\left|\tilde{I}_{2}(t, x)\right| \lesssim \begin{cases}|x|^{\theta-n} & \left|x t^{-\frac{\alpha}{2}}\right| \leq 1 \\ C t^{\frac{\alpha}{2}(\theta+\sigma)}|x|^{-n-\sigma} & \left|x t^{-\frac{\alpha}{2}}\right| \geq 1\end{cases}
$$

where $\sigma=\frac{\theta \alpha-n}{2-\alpha}$.

In particular, when $\left|x t^{-\frac{\alpha}{2}}\right| \geq 1$ with $0<t<1$, we have

$$
t^{\frac{\alpha}{2}(\theta+\sigma)}|x|^{-n-\sigma} \leq t^{\frac{\alpha}{2}(\theta-n)}
$$


thus the following is also valid,

$$
\left|I_{2}(t, x)\right| \sim\left|\tilde{I}_{2}(t, x)\right| \leq \begin{cases}C \max \left\{1,|x|^{\theta-n}\right\}, & |x| \leq t^{\frac{\alpha}{2}} \\ C \max \left\{1, t^{\frac{\alpha}{2}(\theta-n)}\right\}, & t^{\frac{\alpha}{2}} \leq|x| \leq 1 \\ C|x|^{-n-\sigma}, & |x| \geq 1\end{cases}
$$

With the help of (3.22) and (3.28), when $0<t<1$, we get that if $\theta>\theta_{S_{\alpha}}$, then

$$
\left|J^{\theta} S_{\alpha}(t, x)\right| \leq \begin{cases}C \max \left\{1,|x|^{\theta-n}\right\}, & |x| \leq t^{\frac{\alpha}{2}} \\ C \max \left\{1, t^{\frac{\alpha}{2}(\theta-n)}\right\}, & t^{\frac{\alpha}{2}} \leq|x| \leq 1, \\ C|x|^{-n-\sigma}, & |x| \geq 1 .\end{cases}
$$

where $\sigma=\frac{\theta \alpha-n}{2-\alpha}>0$. In particular, if $0<t \leq 1, J^{\theta} S_{\alpha}(t, x)$ is in $L^{1}\left(\mathbb{R}^{n} \backslash B(0,1)\right)$ uniformly.

Case $(b)$ : when $t>1$.

It is easy to notice that the inequality (3.26) is still valid when $t>1$. Then we only need to estimate $I_{2}(t, x)$. We can rewrite $I_{2}(t, x)$ as

$$
\begin{aligned}
I_{2}(t, x)= & \frac{1}{(2 \pi)^{n / 2}} t^{-\alpha n / 2} \int_{\mathbb{R}^{n}} e^{i x t^{-\frac{\alpha}{2}} \cdot \xi}\left(1+t^{-\alpha}|\xi|^{2}\right)^{-\frac{\theta}{2}} E_{\alpha, 1}\left(i^{-\alpha}|\xi|^{2}\right)(1-\Phi(\xi)) \mathrm{d} \xi \\
= & \frac{1}{(2 \pi)^{n / 2}} t^{-\alpha n / 2} \int_{1 \leq|\xi| \leq t^{\alpha / 2}} e^{i x t^{-\frac{\alpha}{2}} \cdot \xi}\left(1+t^{-\alpha}|\xi|^{2}\right)^{-\frac{\theta}{2}} E_{\alpha, 1}\left(i^{-\alpha}|\xi|^{2}\right)(1-\Phi(\xi)) \mathrm{d} \xi \\
& +\frac{1}{(2 \pi)^{n / 2}} t^{-\alpha n / 2} \int_{|\xi| \geq t^{\alpha / 2}} e^{i x t^{-\frac{\alpha}{2}} \cdot \xi}\left(1+t^{-\alpha}|\xi|^{2}\right)^{-\frac{\theta}{2}} E_{\alpha, 1}\left(i^{-\alpha}|\xi|^{2}\right)(1-\Phi(\xi)) \mathrm{d} \xi \\
= & : I_{21}+I_{22} .
\end{aligned}
$$

When $t>1$ and $t^{-\alpha}|\xi|^{2} \leq 1$, for $I_{21}$, we have

$$
\left|I_{21}(t, x)\right| \lesssim t^{-\alpha n / 2}\left(t^{\alpha n / 2}+1\right) \lesssim 1 .
$$

As for $I_{22}(t, x)$, when $t^{-\alpha}|\xi|^{2} \geq 1,\left(1+t^{-\alpha}|\xi|^{2}\right)^{-\frac{\theta}{2}} \sim t^{\alpha \theta / 2}|\xi|^{-\theta}$, then we have that

$$
\left|I_{22}(t, x)\right| \sim\left|\tilde{I}_{2}(t, x)\right| \lesssim \begin{cases}|x|^{\theta-n} & \left|x t^{-\frac{\alpha}{2}}\right| \leq 1 \\ C t^{\frac{\alpha}{2}(\theta+\sigma)}|x|^{-n-\sigma} & \left|x t^{-\frac{\alpha}{2}}\right| \geq 1\end{cases}
$$

Therefore, when $t>1$, we have

$$
\left|J^{\theta} S_{\alpha}(t, x)\right| \leq \begin{cases}C \max \left\{1,|x|^{\theta-n}\right\}, & |x| \leq t^{\frac{\alpha}{2}} \\ C t^{\frac{\alpha}{2}(\theta+\sigma)}|x|^{-n-\sigma}, & |x| \geq t^{\frac{\alpha}{2}}\end{cases}
$$

This finishes the proof.

Corollary 3.5. For any fixed $t_{0}>0$, we have

(1) if $\theta>\theta_{S_{\alpha}}$, then there exist a constant $C>0$ such that for all $t \in\left[\frac{t_{0}}{2}, \frac{3 t_{0}}{2}\right]$,

$$
\left\|J^{\theta} S_{\alpha}(t, x)\right\|_{L^{1}\left(\mathbb{R}^{n}\right)} \leq C .
$$

(2) if $\theta>\theta_{Q_{\alpha}}$, then there exist a constant $C>0$ such that for all $t \in\left[\frac{t_{0}}{2}, \frac{3 t_{0}}{2}\right]$,

$$
\left\|J^{\theta} Q_{\alpha}(t, x)\right\|_{L^{1}\left(\mathbb{R}^{n}\right)} \leq C .
$$

(3) if $\theta>\theta_{P_{\alpha}}$, then hen there exist a constant $C>0$ such that for all $t \in$ $\left[\frac{t_{0}}{2}, \frac{3 t_{0}}{2}\right]$

$$
\left\|J^{\theta} P_{\alpha}(t, x)\right\|_{L^{1}\left(\mathbb{R}^{n}\right)} \leq C
$$


Proof. As in the earlier two proofs, we only prove that the statement is valid for $J^{\theta} S_{\alpha}(t, x)$, as the other cases are similarly proven. If $0<t_{0} \leq 2$, define

$$
M_{t_{0}}(x)= \begin{cases}C \max \left\{1,|x|^{\theta-n}\right\}, & |x| \leq\left(\frac{t_{0}}{2}\right)^{\frac{\alpha}{2}}, \\ C \max \left\{1, t_{0}^{\frac{\alpha}{2}(\theta-n)}\right\}, & \left(\frac{t_{0}}{2}\right)^{\frac{\alpha}{2}} \leq|x| \leq 1, \\ C|x|^{-n-\sigma}, & |x| \geq 1,\end{cases}
$$

and if $t_{0} \geq 2$, define

$$
M_{t_{0}}(x)= \begin{cases}C \max \left\{1,|x|^{\theta-n}\right\}, & |x| \leq\left(\frac{t_{0}}{2}\right)^{\frac{\alpha}{2}} \\ C t_{0}^{\theta+\sigma}|x|^{-n-\sigma}, & |x| \geq\left(\frac{t_{0}}{2}\right)^{\frac{\alpha}{2}}\end{cases}
$$

where $\sigma=\frac{\theta \alpha-n}{2-\alpha}>0$. In either case, $M_{t_{0}}(x) \in L^{1}\left(\mathbb{R}^{n}\right)$. By the estimates (3.15) and (3.16), for any $t \in\left[\frac{t_{0}}{2}, \frac{3 t_{0}}{2}\right],\left|J^{\theta} S_{\alpha}(t, x)\right| \leq M_{t_{0}}(x)$. Thus there exist a constant $C>O$ such that $\left\|J^{\theta} S_{\alpha}(t, x)\right\|_{L^{1}\left(\mathbb{R}^{n}\right)} \leq C$ for all $t \in\left[\frac{t_{0}}{2}, \frac{3 t_{0}}{2}\right]$.

\section{THE PROOF OF THE MAIN THEOREM}

In this section, we prove Theorem 1.1. We divide our proof into three main subsections, i.e. space regularity, time regularity and pointwise convergence to the initial data.

4.1. Space regularity. In this subsection, by exploring the multiplier properties for the corresponding Mittag-Leffler functions, we prove the space regularity for the mild solution. To start with, we give some estimates for the derivatives of the oscillatory Mittag-Leffler functions.

Lemma 4.1. Let $1<\alpha<2$, and assume that $\gamma$ is a multi-index nonnegative integer, then

(1) when $|\xi| \leq 1, D^{\gamma} \widehat{S}_{\alpha}(\xi), D^{\gamma} \widehat{Q}_{\alpha}(\xi)$ and $D^{\gamma} \widehat{P}_{\alpha}(\xi)$ are bounded;

(2) when $|\xi| \geq 1$, we have

(a) $\left|D^{\gamma} \widehat{S}_{\alpha}(\xi)\right| \leq C|\xi|^{\left(\frac{2}{\alpha}-1\right)|\gamma|}$;

(b) $\left|D^{\gamma} \widehat{Q}_{\alpha}(\xi)\right| \leq C|\xi|^{-\frac{2}{\alpha}}|\xi|^{\left(\frac{2}{\alpha}-1\right)|\gamma|}$;

(c) $\left|D^{\gamma} \widehat{P}_{\alpha}(\xi)\right| \leq C|\xi|^{-\frac{2(\alpha-1)}{\alpha}}|\xi|^{\left(\frac{2}{\alpha}-1\right)|\gamma|}$.

Proof. Case 1: When $|\xi| \leq 1$, since the Mittag-Leffler function $E_{\alpha, 1}\left(i^{-\alpha}|\xi|^{2}\right)$ is an entire function, so all its derivatives are bounded.

Case 2: When $|\xi|>1$, let us denote $z=i^{-1}|\xi|^{\frac{2}{\alpha}}$. By induction, it is easy to verify that

$$
\left|D^{\gamma} z\right| \leq C_{\gamma}|\xi|^{\frac{2}{\alpha}-|\gamma|} .
$$

Notice that when $1<\alpha<2$, we have $\frac{2}{\alpha}-1>0$, which implies that $|\xi|^{\left(\frac{2}{\alpha}-1\right)} \rightarrow \infty$ is an increasing term, and it is the main term compared with $|\xi|^{-1}$ as $|\xi| \rightarrow \infty$.

(1) Firstly, we explain the result is valid for $\widehat{S}_{\alpha}(\xi)$. Let $\gamma$ be a multi-index. We rewrite $S_{\alpha}(\xi)$ as a function of $z$, that is $\widehat{S}_{\alpha}(\xi)=E_{\alpha, 1}\left(i^{-\alpha}|\xi|^{2}\right)=E_{\alpha, 1}\left(z^{\alpha}\right)$ with $z=i^{-1}|\xi|^{\frac{2}{\alpha}}$. Then we calculate its derivatives by Faá di Bruno's formula Lemma 2.14, and the main term for $D^{\gamma} E_{\alpha, 1}\left(i^{-\alpha}|\xi|^{2}\right)$ is $\frac{d^{|\gamma|}}{d z|\gamma|} E_{\alpha, 1}\left(z^{\alpha}\right) D^{\gamma} z$ as $|\xi| \rightarrow \infty$. By (2.7) and (4.1), we have

$$
\left|D^{\gamma} \widehat{S}_{\alpha}(\xi)\right| \leq C|\xi|^{\left(\frac{2}{\alpha}-1\right)|\gamma|} .
$$


(2) Similarly, we can prove that

$$
\begin{aligned}
\left|D^{\gamma}\left(z E_{\alpha, 2}\left(i^{-\alpha}|\xi|^{2}\right)\right)\right| & \leq|\xi|^{\left(\frac{2}{\alpha}-1\right)|\gamma|}, \\
\left|D^{\gamma}\left(z^{-1}\right)\right| & \leq C|\xi|^{-\frac{2}{\alpha}-|\gamma|} .
\end{aligned}
$$

By the Leibniz rule, we have

$$
\begin{aligned}
D^{\gamma} \widehat{Q}_{\alpha}(\xi) & =D^{\gamma} E_{\alpha, 2}\left(i^{-\alpha}|\xi|^{2}\right)=D^{\gamma}\left(z^{-1} z E_{\alpha, 2}\left(i^{-\alpha}|\xi|^{2}\right)\right) \\
& =\sum_{\eta \leq \gamma}\left(\begin{array}{l}
\gamma \\
\eta
\end{array}\right) D^{\eta}\left(z^{-1}\right) D^{\gamma-\eta}\left(z E_{\alpha, 2}\left(i^{-\alpha}|\xi|^{2}\right)\right) .
\end{aligned}
$$

By (4.2) and (4.3), we have

$$
\left|D^{\gamma} \widehat{Q}_{\alpha}(\xi)\right| \leq C|\xi|^{-\frac{2}{\alpha}}|\xi|^{\left(\frac{2}{\alpha}-1\right)|\gamma|} .
$$

(3) The method is the same for $P_{\alpha}(x)$, thus we omit the details.

Lemma 4.2. Let $m$ be a smooth function and supported in the unit ball. If

$$
\left|D^{\gamma} m(\xi)\right| \leq C,
$$

then $m \in \mathcal{M}\left(C^{\theta}, C^{\theta}\right)$.

Proof. According to Lemma 2.8, we only need to prove that $K_{m}(x):=\mathcal{F}^{-1}(m(\xi)) \in$ $B_{1, \infty}^{0}$. Since $m$ is a Schwartz function, $K_{m}(x) \in \mathcal{S} \subset B_{1, \infty}^{0}$.

Now we are ready to give the multiplier proposition.

Proposition 4.3. $\quad$ (1) $\widehat{S}_{\alpha}(\xi)$ is a Fourier multiplier from $C^{\frac{n}{\alpha}+s}\left(\mathbb{R}^{n}\right)$ to $C^{s}\left(\mathbb{R}^{n}\right)$;

(2) $\widehat{Q}_{\alpha}(\xi)$ is a Fourier multiplier from $C^{\frac{n}{\alpha}-\frac{2}{\alpha}+s}\left(\mathbb{R}^{n}\right)$ to $C^{s}\left(\mathbb{R}^{n}\right)$; and

(3) $\widehat{P}_{\alpha}(\xi)$ is a Fourier multiplier from $C^{\frac{n}{\alpha}+\frac{2}{\alpha}-2+s}\left(\mathbb{R}^{n}\right)$ to $C^{s}\left(\mathbb{R}^{n}\right)$.

In addition, these results are sharp, in the sense that the operators do not map into $C^{s^{\prime}}\left(\mathbb{R}^{n}\right)$ for any $s^{\prime}>s$.

Proof. The proof for $\widehat{S}_{\alpha}(\xi), \widehat{Q}_{\alpha}(\xi), \widehat{P}_{\alpha}(\xi)$ are similar, thus we only give the details for $\widehat{S}_{\alpha}(\xi)$.

Let $\Phi$ be a smooth function supported in the unit ball, then by Lemma 4.1 , we have

$$
\left|D^{\gamma} \widehat{S}_{\alpha}(\xi) \Phi(\xi)\right| \leq C,
$$

and

$$
\left|D^{\gamma}\left(\widehat{S}_{\alpha}(\xi)(1-\Phi(\xi))\right)\right| \leq C|\xi|^{\left(\frac{2}{\alpha}-1\right)|\gamma|} .
$$

Due to Theorem 2.13, we know that $\widehat{S}_{\alpha}(\xi)(1-\Phi(\xi))$ is a multiplier from $C^{\frac{n}{\alpha}+s}\left(\mathbb{R}^{n}\right)$ to $C^{s}\left(\mathbb{R}^{n}\right)$. Due to Lemma 4.2, we know $\widehat{S}_{\alpha}(\xi) \Phi(\xi)$ is a multiplier from $C^{\frac{n}{\alpha}+s}\left(\mathbb{R}^{n}\right)$ to $C^{\frac{n}{\alpha}+s}\left(\mathbb{R}^{n}\right)$. Collecting these two facts give the desired result.

Next we illustrate that our index is sharp: assume that $\widehat{S}_{\alpha}(\xi)$ is a Fourier multiplier from $C^{\frac{n}{\alpha}+s}\left(\mathbb{R}^{n}\right)$ to $C^{s+\epsilon_{0}}\left(\mathbb{R}^{n}\right)$ for some $\epsilon_{0}>0$. Since $\widehat{S}_{\alpha}(\xi) \Phi(\xi)$ defines an operator from $C^{\frac{n}{\alpha}+s}\left(\mathbb{R}^{n}\right)$ to $C^{\frac{n}{\alpha}+s}\left(\mathbb{R}^{n}\right)$, then we deduce that $\widehat{S}_{\alpha}(\xi)(1-\Phi(\xi))$ is a multiplier from $C^{\frac{n}{\alpha}+s}\left(\mathbb{R}^{n}\right)$ to $C^{s+\epsilon_{0}}\left(\mathbb{R}^{n}\right)$. Let $R(\xi)=\left(\widehat{S}_{\alpha}(\xi)-\frac{1}{\alpha} e^{i|\xi|^{\frac{n}{\alpha}}}\right)(1-\Phi(\xi))$. Then we have $R(\xi) \leq \frac{C}{1+|\xi|^{2}}$, so $R(\xi)$ is a Fourier multiplier from $C^{\frac{n}{\alpha}+s}\left(\mathbb{R}^{n}\right)$ to 
$C^{\frac{n}{\alpha}+s}\left(\mathbb{R}^{n}\right)$. Thus we get $\frac{1}{\alpha} e^{i|\xi|^{\frac{n}{\alpha}}}(1-\Phi(\xi))$ is $C^{\frac{n}{\alpha}+s}\left(\mathbb{R}^{n}\right)$ to $C^{s+\epsilon_{0}}\left(\mathbb{R}^{n}\right)$, which is in contradiction with Theorem 4.4 of Miyachi [25].

The results for $\widehat{Q}_{\alpha}(\xi)$ and $\widehat{P}_{\alpha}(\xi)$ are similarly proven.

For Mittag-Leffler kernels, we have the following scaling properties by changing variables,

$$
\begin{aligned}
& S_{\alpha}(t, x)=t^{-\frac{\alpha}{2} n} S_{\alpha}\left(1, t^{-\frac{\alpha}{2}} x\right), \\
& Q_{\alpha}(t, x)=t^{-\frac{\alpha}{2} n+1} Q_{\alpha}\left(1, t^{-\frac{\alpha}{2}} x\right), \\
& P_{\alpha}(t, x)=t^{-\frac{\alpha}{2} n+\alpha-1} P_{\alpha}\left(1, t^{-\frac{\alpha}{2}} x\right) .
\end{aligned}
$$

therefore we have proven: if $u_{0}(x) \in C^{\frac{n}{\alpha}+s}\left(\mathbb{R}^{n}\right), u_{1}(x) \in C^{\frac{n}{\alpha}-\frac{2}{\alpha}+s}\left(\mathbb{R}^{n}\right)$ and $f(t, x) \in$ $C^{\frac{n+2}{\alpha}-2+s}\left(\mathbb{R}^{n}\right)$ for any $t>0$, then we have $u(t, x) \in C^{s}\left(\mathbb{R}^{n}\right)$ for every $t>0$.

4.2. Time regularity. In this subsection, we will demonstrate that, if $u_{0} \in C^{\frac{n}{\alpha}+\epsilon}\left(\mathbb{R}^{n}\right)$, $u_{1} \in C^{2+\frac{n}{\alpha}-\frac{2}{\alpha}+\epsilon}\left(\mathbb{R}^{n}\right)$ and $f \in C_{t}^{0}\left((0, \infty): C^{\frac{n}{\alpha}+\frac{2}{\alpha}+\epsilon}\left(\mathbb{R}^{n}\right)\right)$, then $J^{2-\alpha} u^{\prime}$ is continuously differentiable in time for $t>0$.

In order to do that, notice if the initial data is regular enough, then we can write $\frac{\partial}{\partial t} I_{t}^{2-\alpha} u^{\prime}$ as three terms

$$
\frac{\partial}{\partial t} I_{t}^{2-\alpha} u^{\prime}=\frac{\partial}{\partial t} I_{t}^{2-\alpha} u_{0}^{\prime}(t, x)+\frac{\partial}{\partial t} I_{t}^{2-\alpha} u_{1}^{\prime}(t, x)+\frac{\partial}{\partial t} I_{t}^{2-\alpha} u_{f}^{\prime}(t, x),
$$

where

$$
\begin{aligned}
\frac{\partial}{\partial t} I_{t}^{2-\alpha} u_{0}^{\prime}(t, x) & =\int_{\mathbb{R}^{n}} M_{\alpha}(t, x-y) u_{0}(y) \mathrm{d} y, \\
\frac{\partial}{\partial t} I_{t}^{2-\alpha} u_{1}^{\prime}(t, x) & =\int_{\mathbb{R}^{n}} N_{\alpha}(t, x-y) u_{1}(y) \mathrm{d} y, \\
\frac{\partial}{\partial t} I_{t}^{2-\alpha} u_{f}^{\prime}(t, x) & =f(t, x)+\int_{0}^{t} \int_{\mathbb{R}^{n}} L_{\alpha}(t-\tau, x-y) f(\tau, y) \mathrm{d} y \mathrm{~d} \tau,
\end{aligned}
$$

with

$$
\begin{aligned}
& \widehat{M_{\alpha}}(t, \xi)=i^{-\alpha}|\xi|^{2} E_{\alpha, 1}\left(i^{-\alpha} t^{\alpha}|\xi|^{2}\right), \\
& \widehat{N_{\alpha}}(t, \xi)=t^{1-\alpha} E_{\alpha, 2-\alpha}\left(i^{-\alpha} t^{\alpha}|\xi|^{2}\right) \\
& \widehat{L_{\alpha}}(t, \xi)=i^{-\alpha}|\xi|^{2} t^{\alpha-1} E_{\alpha, \alpha}\left(i^{-\alpha} t^{\alpha}|\xi|^{2}\right) .
\end{aligned}
$$

Therefore if we can verify that

$$
M_{\alpha}(t, \cdot) * u_{0}(x), \quad N_{\alpha}(t, \cdot) * u_{1}(x), \quad \text { and } \quad \int_{0}^{t} \int_{\mathbb{R}^{n}} L_{\alpha}(t-\tau, x-y) f(\tau, y) \mathrm{d} y \mathrm{~d} \tau
$$

exist for every $x \in \mathbb{R}^{n}$ and it is continuous with respect to $t>0$, then we have our desired results.

For the above kernels, we have the following scaling properties.

$$
\begin{aligned}
M_{\alpha}(t, x) & =t^{-\frac{\alpha}{2} n-\alpha} M_{\alpha}\left(1, t^{-\frac{\alpha}{2}} x\right), \\
N_{\alpha}(t, x) & =t^{-\frac{\alpha}{2} n+1-\alpha} N_{\alpha}\left(1, t^{-\frac{\alpha}{2}} x\right), \quad \text { and } \\
L_{\alpha}(t, x) & =t^{-\frac{\alpha}{2} n-1} L_{\alpha}\left(1, t^{-\frac{\alpha}{2}} x\right)
\end{aligned}
$$

Analogously, we write $M_{\alpha}(x)=M_{\alpha}(1, x), N_{\alpha}(x)=N_{\alpha}(1, x)$ and $L_{\alpha}(x)=L_{\alpha}(1, x)$ for short. 
Lemma 4.4. Let $\theta>0$. Then $J^{\theta} M_{\alpha}(x), J^{\theta} N_{\alpha}(x)$ and $J^{\theta} L_{\alpha}(x)$ are all smooth functions throughout $\mathbb{R}^{n} \backslash\{0\}$ and have the following asymptotic behaviors as $|x| \rightarrow$ $\infty$, there exist some constants $A$ and $B$ which depends on $\alpha, \theta$ and $n$, such that,

$$
\begin{aligned}
& J^{\theta} M_{\alpha}(x)=A|x|^{\frac{n \alpha+2 \alpha-n-\alpha \theta}{2-\alpha}} e^{i B|x|^{\frac{2}{\alpha-2}}+o\left(|x|^{\frac{n \alpha+2 \alpha-n-\alpha \theta}{2-\alpha}}\right),} \\
& J^{\theta} N_{\alpha}(x)=A|x|^{\frac{n \alpha+2(\alpha-1)-n-\alpha \theta}{2-\alpha}} e^{i B|x|^{\frac{2}{\alpha-2}}}+o\left(|x|^{\frac{n \alpha+2(\alpha-1)-n-\alpha \theta}{2-\alpha}}\right), \\
& J^{\theta} L_{\alpha}(x)=A|x|^{\frac{n \alpha+2-n-\alpha \theta}{2-\alpha}} e^{i B|x|^{\frac{2}{\alpha-2}}}+o\left(|x|^{\frac{n \alpha+2-n-\alpha \theta}{2-\alpha}}\right),
\end{aligned}
$$

and the following asymptotic behaviors as $|x| \rightarrow 0$, and

$$
\begin{aligned}
\left|J^{\theta} M_{\alpha}(x)\right| & \sim \begin{cases}C, & \text { when } \theta>n, \\
C \log \frac{1}{|x|}, & \text { when } \theta=n, \\
C|x|^{\theta-n}+o\left(|x|^{\theta-n}\right), & \text { when } \theta<n .\end{cases} \\
\left|J^{\theta} N_{\alpha}(x)\right|,\left|J^{\theta} L_{\alpha}(x)\right| & \sim \begin{cases}C, & \text { when } \theta>n-2, \\
C \log \frac{1}{\mid x}, & \text { when } \theta=n+2, \\
C|x|^{2+\theta-n}+o\left(|x|^{2+\theta-n}\right), & \text { when } \theta<n-2 .\end{cases}
\end{aligned}
$$

In addition, if we denote $\theta_{M_{\alpha}}=\frac{n}{\alpha}+2, \theta_{N_{\alpha}}=\frac{n}{\alpha}+2-\frac{2}{\alpha}, \theta_{L_{\alpha}}=\frac{n}{\alpha}+\frac{2}{\alpha}$, then we have

(1) if $\theta>\theta_{M_{\alpha}}$, then $J^{\theta} M_{\alpha}(x) \in L^{1}\left(\mathbb{R}^{n}\right)$;

(2) if $\theta>\theta_{N_{\alpha}}$, then $J^{\theta} N_{\alpha}(x) \in L^{1}\left(\mathbb{R}^{n}\right)$; and

(3) if $\theta>\theta_{L_{\alpha}}$, then $J^{\theta} L_{\alpha}(x) \in L^{1}\left(\mathbb{R}^{n}\right)$.

Proof. The proof is the similar to Lemma 3.1, thus we omit the details.

Lemma 4.5. For any fixed $t_{0}>0$, we have

(1) if $\theta>\theta_{M_{\alpha}}$, then there exist a constant $C>0$ such that for all $t \in\left[\frac{t_{0}}{2}, \frac{3 t_{0}}{2}\right]$,

$$
\left\|J^{\theta} M_{\alpha}(t, x)\right\|_{L^{1}\left(\mathbb{R}^{n}\right)} \leq C .
$$

(2) if $\theta>\theta_{N_{\alpha}}$, then there exist a constant $C>0$ such that for all $t \in\left[\frac{t_{0}}{2}, \frac{3 t_{0}}{2}\right]$,

$$
\left\|J^{\theta} N_{\alpha}(t, x)\right\|_{L^{1}\left(\mathbb{R}^{n}\right)} \leq C .
$$

(3) if $\theta>\theta_{L_{\alpha}}$, then hen there exist a constant $C>0$ such that for all $t \in$ $\left[\frac{t_{0}}{2}, \frac{3 t_{0}}{2}\right]$

$$
\left\|J^{\theta} L_{\alpha}(t, x)\right\|_{L^{1}\left(\mathbb{R}^{n}\right)} \leq C .
$$

Proof. The proof is similar to Lemma 3.4 and omit the details.

Proposition 4.6. Assume that $u_{0} \in C^{\frac{n}{\alpha}+2+\epsilon}\left(\mathbb{R}^{n}\right), u_{1} \in C^{\frac{n}{\alpha}+2-\frac{2}{\alpha}+\epsilon}\left(\mathbb{R}^{n}\right)$ and $f \in$ $C_{t}^{0}\left((0, \infty) ; C^{\frac{n+2}{\alpha}+\epsilon}\left(\mathbb{R}^{n}\right)\right)$, then $I_{t}^{2-\alpha} u^{\prime}(t, x)$ is continuous differentiable with respect to $t>0$.

Proof. If $u_{0} \in C^{\frac{n}{\alpha}+2+\epsilon}\left(\mathbb{R}^{n}\right)$, then $J^{-\left(\frac{n}{\alpha}+2+\epsilon\right)} u_{0}(x) \in L^{\infty}\left(\mathbb{R}^{n}\right) \cap C\left(\mathbb{R}^{n}\right)$. For any fixed $t_{0}>0 J^{\frac{n}{\alpha}+2+\epsilon} M_{\alpha}(t, x) \in L^{1}\left(\mathbb{R}^{n}\right)$ for all $t \in\left[t_{0} / 2,3 t_{0} / 2\right]$. By the dominated convergence theorem, we can change the limit and the integral, thus the following equality is valid,

$$
\left.\frac{\partial}{\partial t} I_{t}^{2-\alpha} u_{0}^{\prime}(t, x)\right|_{t=t_{0}}=J^{\frac{n}{\alpha}+2+\epsilon} M_{\alpha}\left(t_{0}, \cdot\right) * J^{-\frac{n}{\alpha}+2+\epsilon} u_{0}(x)=M_{\alpha}\left(t_{0}, \cdot\right) * u_{0}(x),
$$


thus $\frac{\partial}{\partial t} I_{t}^{2-\alpha} u_{0}^{\prime}(t, x)$ is continuous at every $t_{0}>0$.

That is, for all $x \in \mathbb{R}^{n}, I_{t}^{2-\alpha} u_{0}^{\prime}(t, x)$ is continuously differentiable with respect to $t>0$. Similarly, $x \in \mathbb{R}^{n}, I_{t}^{2-\alpha} u_{1}^{\prime}(t, x)$ and $I_{t}^{2-\alpha} u_{f}^{\prime}(t, x)$ is continuously differentiable with respect to $t>0$. Thus the statement is valid.

Conclusion: If $u_{0}(x) \in C^{\frac{n}{\alpha}+2+\epsilon}\left(\mathbb{R}^{n}\right), u_{1}(x) \in C^{\frac{n}{\alpha}+2-\frac{2}{\alpha}++\epsilon}\left(\mathbb{R}^{n}\right)$ and $f \in C_{t}^{0}\left((0, \infty) ; C^{\frac{n}{\alpha}+\frac{2}{\alpha}+\epsilon}\left(\mathbb{R}^{n}\right)\right)$, then for each $x \in \mathbb{R}^{n}, I_{t}^{2-\alpha} u^{\prime}(t, x)$ is continuously differentiable with respect to $t$.

4.3. Pointwise convergence to the initial data. In this subsection, we restrict the time $t$ to $0<t<1$, since we only discuss the behavior $u(t, x)$ as $t \rightarrow 0$. In order to demonstrated the validity of the pointwise convegence, we give the following 'almost finite speed propagation' property. Let us denote $\Phi_{R}(x)$ be a smooth function equal to 1 in $B(0, R)$ and 0 in $B(0, R+1)^{c}$.

Lemma 4.7 (Mismatch estimates). Assume that $0<t<1$, and $\varphi(x) \in C^{\theta}\left(\mathbb{R}^{n}\right)$.

(1) If $\theta>\theta_{S_{\alpha}}$, then for any $\delta>0$, there exist a number $R_{\delta}>1$, such that

$\left\|\chi_{B(0,1)}(x) \int_{\mathbb{R}^{n}} J^{\theta} S_{\alpha}(t, x-y)\left(1-\Phi_{R_{\delta}}(y)\right) J^{-\theta} \varphi(y) \mathrm{d} y\right\|_{L^{\infty}\left(\mathbb{R}^{n}\right)} \leq \delta \|\left. J^{-\theta} \varphi(x)\right|_{L^{\infty}\left(\mathbb{R}^{n}\right)}$.

(2) If $\theta>\theta_{Q_{\alpha}}$, then for any $\delta>0$, there exist a number $R_{\delta}>1$, such that

$\left\|\chi_{B(0,1)}(x) \int_{\mathbb{R}^{n}} J^{\theta} Q_{\alpha}(t, x-y)\left(1-\Phi_{R_{\delta}}(y)\right) J^{-\theta} \varphi(y) \mathrm{d} y\right\|_{L^{\infty}\left(\mathbb{R}^{n}\right)} \leq \delta \|\left. J^{-\theta} \varphi(x)\right|_{L^{\infty}\left(\mathbb{R}^{n}\right)}$.

(3) If $\theta>\theta_{P_{\alpha}}$, then for any $\delta>0$, there exist a number $R_{\delta}>1$, such that

$\left\|\chi_{B(0,1)}(x) \int_{\mathbb{R}^{n}} J^{\theta} P_{\alpha}(t, x-y)\left(1-\Phi_{R_{\delta}}(y)\right) J^{-\theta} \varphi(y) \mathrm{d} y\right\|_{L^{\infty}\left(\mathbb{R}^{n}\right)} \leq \delta \|\left. J^{-\theta} \varphi(x)\right|_{L^{\infty}\left(\mathbb{R}^{n}\right)}$.

Proof. We give the proof for $S_{\alpha}(t, x)$, the others are analogous. By Lemma 3.4 $J^{\theta} S_{\alpha}(t, x) \in L^{1}\left(\mathbb{R}^{n} \backslash B(0,1)\right)$ for all $0<t<1$. That is, for any $\delta>0$, there exist a number $R_{\delta}>1$ big enough such that for any $0<t<1$,

$$
\int_{|x| \geq R_{\delta}-1}\left|J^{\theta} S_{\alpha}(t, x)\right| \mathrm{d} x \leq \delta .
$$

When $\varphi(x) \in C^{\theta}\left(\mathbb{R}^{n}\right)$, we have

$$
\left\|\left(\left(1-\Phi_{R_{\delta}}(x)\right) J^{-\theta} \varphi(x)\right)\right\|_{L^{\infty}\left(\mathbb{R}^{n}\right)} \leq\left\|J^{-\theta} \varphi(x)\right\|_{L^{\infty}\left(\mathbb{R}^{n}\right)}<\infty .
$$

Since we have $x \in B(0,1), y \in B\left(0, R_{\delta}\right)^{c}$, then $x-y \in B\left(0, R_{\delta}-1\right)^{c}$, thus

$$
\begin{aligned}
& \left|\chi_{B(0,1)}(x) \int_{\mathbb{R}^{n}} J^{\theta} S_{\alpha}(t, x-y)\left(1-\Phi_{R_{\delta}}(y)\right) J^{-\theta} \varphi(y) \mathrm{d} y\right| \\
\leq & \left\|\left(\left(1-\Phi_{R_{\delta}}\right)(y) J^{-\theta} \varphi(y)\right)\right\|_{L^{\infty}\left(\mathbb{R}^{n}\right)} \int_{|y| \geq R_{\delta}-1}\left|J^{\theta} S_{\alpha}(t, y)\right| \mathrm{d} y \\
\leq & \delta\left\|J^{-\theta} \varphi(x)\right\|_{L^{\infty}\left(\mathbb{R}^{n}\right)} .
\end{aligned}
$$

Thus we proved the lemma.

Proposition 4.8. If $u_{0} \in C^{2+\frac{n}{\alpha}+\epsilon}\left(\mathbb{R}^{n}\right), u_{1} \in C^{2+\frac{n}{\alpha}-\frac{2}{\alpha}+\epsilon}\left(\mathbb{R}^{n}\right)$ and $f \in C_{t}^{0}\left((0, \infty) ; C^{\frac{n}{\alpha}+\frac{2}{\alpha}+\epsilon}\left(\mathbb{R}^{n}\right)\right)$, then the mild solution $u(t, x)$ pointwise convergence to the initial data, that is $\lim _{t \rightarrow 0} u(t, x)=u_{0}(x)$ and $\lim _{t \rightarrow 0} \partial_{t} u(t, x)=u_{1}(x)$. 
Proof. It is easy to notice that $\widehat{S}_{\alpha}(t, \xi), \widehat{Q}_{\alpha}(t, \xi), \widehat{P}_{\alpha}(t, \xi)$ are continuous function at $t=0$, and $\widehat{S}_{\alpha}(0, \xi)=1, \widehat{Q}_{\alpha}(0, \xi)=0, \widehat{P}_{\alpha}(0, \xi)=0$.

Step 1: First we claim that if $J^{-\theta} u_{0}$ has compact support, the pointwise convergence is valid. This is because if $J^{-\theta} u_{0} \in C_{c}^{0}\left(\mathbb{R}^{n}\right)$ with $\theta>\frac{n}{\alpha}>\frac{n}{2}$, then $u_{0} \in H^{\theta}\left(\mathbb{R}^{n}\right) \hookrightarrow L^{\infty}\left(\mathbb{R}^{n}\right)$. Also we have

$$
\left|\widehat{u}_{0}(t, \xi)\right|=\left|\widehat{u}_{0}(\xi) E_{\alpha}\left(i^{-\alpha} t^{\alpha}|\xi|^{2}\right)\right| \leq C\left|\widehat{u}_{0}(\xi)\right|,
$$

thus, $u_{0}(t, x) \in H^{\theta}\left(\mathbb{R}^{n}\right) \hookrightarrow L^{\infty}\left(\mathbb{R}^{n}\right)$. Therefore,

$$
\begin{aligned}
\left\|u_{0}(t, x)-u_{0}(x)\right\|_{L^{\infty}\left(\mathbb{R}^{n}\right)} & \leq\left\|u_{0}(t, x)-u_{0}(x)\right\|_{H^{\theta}\left(\mathbb{R}^{n}\right)} \\
& \leq\left\|\left(E_{\alpha}\left(i^{-\alpha} t^{\alpha}|\xi|^{2}\right)-1\right)\left(1+4 \pi|\xi|^{2}\right)^{\theta / 2} \widehat{u}_{0}(\xi)\right\|_{L^{2}} \\
& \rightarrow 0 \text { as } t \rightarrow 0 .
\end{aligned}
$$

In other words, if we assume that $\operatorname{supp}\left(J^{-\theta} u_{0}\right) \subset B(0, R)$ for a fixed $R>0$. Then for any $\delta>0$, there exist $0<t_{\delta}<1$ such that for any $0<t<t_{\delta}$ and $x \in \mathbb{R}^{n}$, we have

$$
\begin{aligned}
\left|u_{0}(t, x)-u_{0}(x)\right| & =\left|\int_{\mathbb{R}^{n}} J^{\theta} S_{\alpha}(t, x-y) \Phi_{R}(y) J^{-\theta} u_{0}(y) \mathrm{d} y-J^{\theta}\left(\Phi_{R}(x) J^{-\theta} u_{0}(x)\right)\right| \\
& \leq \frac{\delta}{2}
\end{aligned}
$$

Step 2: For general $u_{0} \in C^{\theta}\left(\mathbb{R}^{n}\right)$ with $\theta>\frac{n}{\alpha}$, we only need to prove that for any $x \in B(0,1), u_{0}(t, x) \rightarrow u_{0}(x)$, which is equivalent to $\chi_{B(0,1)} u_{0}(t, x) \rightarrow \chi_{B(0,1)} u_{0}(x)$. For any $\delta>0$, due to Lemma 4.7 and the integrability of $G^{\theta}(x)$, there exist a $R_{\delta}>1$ such that for any $0<t<1$,

$$
\left|\chi_{B(0,1)}(x) \int_{\mathbb{R}^{n}} J^{\theta} S_{\alpha}(t, x-y)\left(1-\Phi_{R_{\delta}}(y)\right) J^{-\theta} u_{0}(y) \mathrm{d} y\right| \leq \frac{\delta}{2},
$$

and

$$
\left.\mid \chi_{B(0,1)}(x) \int_{\mathbb{R}^{n}} G^{\theta}(x-y)\left(1-\Phi_{R_{\delta}}(y)\right) J^{-\theta} u_{0}(y)\right) \mathrm{d} y \mid \leq \frac{\delta}{4} .
$$

For the above $R_{\delta}$, since $\Phi_{R_{\delta}} J^{-\theta} u_{0}$ is compactly supported, then by the step 1 , there exists $t_{\delta}$ such that for any $0<t<t_{\delta}$, we have

$$
\left|\int_{\mathbb{R}^{n}} J^{\theta} S_{\alpha}(t, x-y)\left(\Phi_{R_{\delta}}(y) J^{-\theta} u_{0}(y)\right) \mathrm{d} y-J^{\theta}\left(\Phi_{R_{\delta}}(x) J^{-\theta} u_{0}(x)\right)\right| \leq \frac{\delta}{4} .
$$


Combing (4.17), (4.18) and (4.19), we have

$$
\begin{aligned}
&\left.\mid \chi_{B(0,1)}(x) u_{0}(t, x)-\chi_{B(0,1)}(x) u_{0}(x)\right) \mid \\
&= \mid \chi_{B(0,1)}(x) \int_{\mathbb{R}^{n}} J^{\theta} S_{\alpha}(t, x-y)\left(\Phi_{R_{\delta}}(y) J^{-\theta} u_{0}(y)\right) \mathrm{d} y \\
&+\chi_{B(0,1)}(x) \int_{\mathbb{R}^{n}} J^{\theta} S_{\alpha}(t, x-y)\left(\left(1-\Phi_{R_{\delta}}(y)\right) J^{-\theta} u_{0}(y)\right) \mathrm{d} y \\
&-\chi_{B(0,1)}(x) J^{\theta}\left(\Phi_{R_{\delta}}(x) J^{-\theta} u_{0}(x)\right)-\chi_{B(0,1)}(x) J^{\theta}\left(\left(1-\Phi_{R_{\delta}}(x)\right) J^{-\theta} u_{0}(x)\right) \mid \\
& \leq\left|\chi_{B(0,1)}(x)\left(\int_{\mathbb{R}^{n}} J^{\theta} S_{\alpha}(t, x-y)\left(\Phi_{R_{\delta}}(y) J^{-\theta} u_{0}(y)\right) \mathrm{d} y-J^{\theta}\left(\Phi_{R_{\delta}}(x) J^{-\theta} u_{0}(x)\right)\right)\right| \\
&+\left|\chi_{B(0,1)}(x) \int_{\mathbb{R}^{n}} J^{\theta} S_{\alpha}(t, x-y)\left(\left(1-\Phi_{R_{\delta}}(y)\right) J^{-\theta} u_{0}(y)\right) \mathrm{d} y\right| \\
&+\left|\chi_{B(0,1)}(x) J^{\theta}\left(\left(1-\Phi_{R_{\delta}}(x)\right) J^{-\theta} u_{0}(x)\right)\right| \\
& \leq \frac{\delta}{4}+\frac{\delta}{2}+\frac{\delta}{4}=\delta . \\
& \text { that is } \lim _{t \rightarrow 0} u_{0}(t, x)=u_{0}(x) \text { for any } x \in B(0,1) .
\end{aligned}
$$

Similarly, we can prove that $\lim _{t \rightarrow 0} u_{1}(t, x)=0$ and $\lim _{t \rightarrow 0} u_{f}(t, x)=0$ under our assumptions. Therefore

$$
\lim _{t \rightarrow 0} u(t, x)=u_{0}(x)
$$

Step 3: By direct calculation, we have

$$
\partial_{t} u(t, x)=P_{\alpha}(t, x) * u_{0}(x)+S_{\alpha}(t, x) * u_{1}(t, x)+\int_{0}^{t} \widehat{H}(t-\tau, \xi) \widehat{f}(\tau, \xi) \mathrm{d} \xi,
$$

where $\widehat{H}(t-\tau, \xi)=i^{-\alpha} t^{\alpha-2} E_{\alpha, \alpha-1}\left(i^{-\alpha} t^{\alpha}|\xi|^{2}\right)$. Notice that $\widehat{H}(t-\tau, \xi)$ is locally $L^{1}$ as a function of $t$, using the same argument in step 1 , we can prove that under our assumption,

$$
\lim _{t \rightarrow 0} \partial_{t} u(t, x)=u_{1}(x)
$$

Thus we verifies the proposition.

\section{The SchröDinger EQUATION}

In this section, we discuss the Hölder regularity for the Schrödinger equation and demonstrate that Theorem 1.2 is valid.

Definition 5.1. The mild solution (1.3) is given by

$$
\begin{aligned}
u(t, x) & =S_{1}(t, \cdot) * u_{0}(x)+\int_{0}^{t} \int_{\mathbb{R}^{n}} S_{1}(t-\tau, x-y) f(\tau, y) \mathrm{d} y \mathrm{~d} \tau \\
& :=u_{0}(t, x)+u_{f}(t, x),
\end{aligned}
$$

where $\widehat{S}_{1}(t, \xi)=e^{-i|\xi|^{2} t}$, as long as the integral exist.

Lemma 5.2. $\widehat{S}_{1}(\xi)$ is a multiplier from $C^{n+s}\left(\mathbb{R}^{n}\right)$ to $C^{s}\left(\mathbb{R}^{n}\right)$.

Proof. We split $\widehat{S}_{1}(\xi)$ into high frequency part and low frequency part. For the high frequency part, it is a multiplier from $C^{n+s}\left(\mathbb{R}^{n}\right)$ to $C^{s}\left(\mathbb{R}^{n}\right)$ by Theorem 4.4 in [25. And the low frequency part is a multiplier from $C^{n+s}\left(\mathbb{R}^{n}\right)$ to $C^{s+n}\left(\mathbb{R}^{n}\right)$ by Lemma 4.2, Thus we arrive at this statement. 
For the Schrödinger equation, using the similar estimates in Lemma 3.4 for $J^{\theta} S_{1}(t, x)$ and $J^{\theta} \partial_{t} S_{1}(t, x)$, we can prove that when $\theta>\theta_{S_{1}}=n, J^{\theta} S_{1}(t, x) \in$ $L^{1}\left(\mathbb{R}^{n}\right)$ uniformly in $0<t<1$ and when $\theta>n+2, J^{\theta} \partial_{t} S_{1}(t, x) \in L^{1}\left(\mathbb{R}^{n}\right)$ uniformly in $0<t<1$. Therefore when $u_{0}(x) \in C^{n+2+\epsilon}\left(\mathbb{R}^{n}\right), u(t, x)$ is continuous differentiable with respect to $t$ for every $x \in \mathbb{R}^{n}$ and it is the pointwise convergence to the initial data using the similar estimates for $J^{\theta} S_{1}(t, x)$. Thus we omit the details. In conclusion, Theorem 1.2 is valid.

Here we give another simpler proof for the pointwise convergence due to the high regularity for the initial data.

Proposition 5.3. If $u_{0}(x) \in C^{n+\epsilon}\left(\mathbb{R}^{n}\right)$ with $\epsilon>0$, the mild solution (5.1) pointwise convergent to the initial date.

Proof. By the dominated convergence theorem, the following equality is valid,

$$
\lim _{t \rightarrow 0} \int_{\mathbb{R}^{n}} e^{i x \cdot \xi} e^{-i|\xi|^{2} t} \frac{\mathrm{d} \xi}{\left(1+|\xi|^{2}\right)^{\frac{n+\epsilon}{2}}}=\int_{\mathbb{R}^{n}} e^{i x \cdot \xi} \frac{\mathrm{d} \xi}{\left(1+|\xi|^{2}\right)^{\frac{n+\epsilon}{2}}}=G^{n+\epsilon}(x),
$$

therefore we have

$$
\begin{aligned}
\lim _{t \rightarrow 0} u_{0}(t, x) & =\lim _{t \rightarrow 0} S_{1}(t, \cdot) * u_{0}(x) \\
& =\lim _{t \rightarrow 0} J^{n+\epsilon} S_{1}(t, \cdot) * J^{-(n+\epsilon)} u_{0}(x) \\
& =G^{n+\epsilon} * J^{-(n+\epsilon)} u_{0}(x)=u_{0}(x) .
\end{aligned}
$$

where we use the fact that $J^{n+\epsilon} S_{1}(t, x)$ is controlled by a integrable function pointwise uniformly for $0<t<1$ to verify the exchange of the integral and the limit. Similarly, we have $\lim _{t \rightarrow 0} u_{f}(t, x)=0$. In this way, we proved the pointwise convergence.

Acknowledgements. Jiqiang Zheng was partially supported by NSFC under grant 11831004 and 11771041.

\section{REFERENCES}

[1] Mark Allen, Luis Caffarelli, Alexis Vasseur, A parabolic problem with a fractional-time derivative, Arch. Ration, Mech, Anal. 221 (2016), 603-630. 1

[2] Albert P. Calderón, Lebesgue spaces of differentiable functions and distributions, Proc. Sympos. Pure Math., 4(1961), 44-49. 7

[3] Gregory M. Constantine, Thomas H. Savits, A multivariate Faá di Bruno's formula with applications, Trans. Amer. Math. Soc., 348(1996), 503-520. 8

[4] Jianping Dong, Mingyu Xu, Space-time fractional Schrödinger equation with time dependent potentials, J. Math. Anal. Appl., 344(2008), 1005-1017. 1

[5] Lawrence C. Evans, Partial Differential Equations, American Mathematical Society, 2012. 2

[6] Yasuhiro Fujita, Integrodifferential equation which interpolates the heat equation and the wave equation, Osaka J. Math. 27 (1990), 309-321. 1

[7] Yasuhiro Fujita, Integrodifferential equation which interpolates the heat equation and the wave equation (II), Osaka J. Math. 27(1990), 797-804. 1

[8] Rudolf Gorenflo, Anatoly A. Kilbas, Francesco Mainarid, Sergei V. Rogosin, Mittag-Leffler functions, Related Topics and Applications, Springer Monographs in Mathematics, SpringerVerlag Berlin Heidelberg, 2014. 5

[9] Ricardo Grande, Space-time fractional nonlinear Schrödinger equation, preprint, arXiv: 1810.07327v1, 2018. 1 
[10] Hitoshi Hirata, Changxing Miao, Space-time estimates of linear flow and application to some nonlinear integro-differential equations corresponding to fractional-order time derivative, Adv. Differential Equations, 7(2002), 217-236. 1]

[11] Lars Höramnder, The Analysis of Linear Partial Differential Operators, Vol. II: Differential Operators with Constant Coefficients, Springer, 2005. 2

[12] Jukka Kemppainen, Juhana Siljander, Rico Zacher, Representation of solutions and largetime behavior for fully nonlocal diffusion equations, J. Differential Equations, 263(2017), 149-201. 2

[13] Rowan Killip, Monica Visan, The defocusing energy-supercritical nonlinear wave equation in three space dimensions, Tran. Amer. Math. Soc., 363(2011), 3893-3934. 2

[14] Ildoo Kim, Kyeong-Hun Kim, Sungbin Lim, An $L_{q}\left(L_{p}\right)$ theory for the time fractional evolution equations with variable coefficients, Adv. Math. 306(2017), 123-176. 1]

[15] Kyeong-Hun Kim, Sungbin Lim, Asymptotic behaviors of fundamental solution and its derivatives related to space-time fractional differential equations, J. Korean Math. Soc., 53(2016), 929-967. 1012

[16] Anatoly N. Kochubei, Asymptotic properties of solutions of the fractional diffusion-wave equation, Frac. Calc. Appl. Anal. 17 (2014), 881-896. 2

[17] Anatoly N. Kochubei, Cauchy problem for fractional diffusion-wave equations with variable coefficients, Applicable analysis 93(2014), 2211-2242. 2

[18] Nick Laskin, Fractional Quantum Mechanics, World Scientific, 2018. 1

[19] Francesco Mainardi, Yuri Luchko, Gianni Pagnini, The fundamental solution of the spacetime fractional diffusion equation, Fract. Calc. Appl. Anal., 4(2001), 153-192. 1

[20] Changxing Miao, Lecture notes on modern harmonic analysis and applications. Monographs on Modern Pure Mathematics, No. 63, Higher Education Press, 2018. 12

[21] Changxing Miao, Han Yang, The self-similar solutions to some nonlinear Integro-differential equations corresponding to fractional order time derivative, Acta Math. Sinica, 21(2005), 13371350. 1

[22] Changxing Miao, Baoquan Yuan, Bo Zhang, Well-posedness of the Cauchy problem for the fractional power disspative equations, NONLINEAR ANAL-THEOR, 68(2008), 461-484. 1 2

[23] Mitchell H. Tailbleson, On the theory of Lipschitz spaces of distributions on Euclidean $n$ space, I. Principal properties, J. Math. Mech. 13(1964), 407-479. 7

[24] Mitchell H. Tailbleson, On the theory of Lipschitz spaces of distributions on Euclidean $n$ space, II. Translation invariant operators, duality and interpolation, J. Math. Mech. 14(1965), 821-839. 6

[25] Akihiko Miyachi, On some singular Fourier multipliers, J. Fac. Sci. Univ. Tokyo Sec. IA 28(1981), 267-315. 3, $7,0,19,23$

[26] Mark Naber, Time fractional Schrödinger equation, J. Math. Phys., 45(2004), 3339-3352. 1

[27] Igor Podlubny, Fractional Differential Equations, Mathematics in Science and Engineering, 1998. 4

[28] Arsen V. Pskhu, The fundamental solution of a diffusion-wave equation of fractional order, Izvestiya: Math. 73(2009), 351-392. 2

[29] Hans Tribel, Theory of Function Spaces, Monographs in Mathematics, 78, 1983. 6

[30] W. R. Schneider, and Walter. Wyss, Fractional diffusion and wave equations, J. Math. Phys., 30(1989), 134-144. 1

[31] Elias M. Stein, Singular Integrals and Differentiability Properties of Functions, Princeton University Press, 1970. 8

[32] Xiaoyan Su, Shiliang Zhao, Miao Li, Dispersive estimates for fractional time and space Schrödinger equation, arXiv.1901.00957v1, to appear in Math. Meth. Appl. Sci. (2019). 1111

[33] Xiaoyan Su, Shiliang Zhao, Miao Li, Local well-posedness of semilinear space-time fractional Schrödinger equation, arXiv:1901.00394v1, to appear in J. Math. Anal. Appl. (2019). 1

Institute of Applied Physics and Computational Mathematics, 100094, P.R.China

E-mail address: suxiaoyan0427@qq.com

Institute of Applied Physics and Computational Mathematics, 100094, P.R.China

E-mail address: zhengjiqiang@gmail.com 